\title{
Systematic review of clinical outcomes in hybrid procedures for aortic arch dissections and other arch diseases
}

\author{
Piergiorgio Cao, MD, FRCS, ${ }^{\mathrm{a}}$ Paola De Rango, MD, PhD, ${ }^{\mathrm{b}}$ Martin Czerny, $\mathrm{MD},{ }^{\mathrm{c}}$ Arturo Evangelista, $\mathrm{MD},{ }^{\mathrm{d}}$ \\ Rossella Fattori, MD, ${ }^{\mathrm{e}}$ Christoph Nienaber, MD ${ }^{\mathrm{f}}$ Hervè Rousseau, MD, ${ }^{\mathrm{g}}$ and Marc Schepens, $\mathrm{MD}^{\mathrm{h}}$
}

Objective: Available data on clinical outcomes of hybrid aortic arch repair are limited, especially for patients with aortic dissection. The objective of this review was to provide pooled analysis of periprocedural mortality and neurologic outcomes in hybrid procedures involving the aortic arch for dissection and other aortic diseases. Methods: Studies involving hybrid aortic arch procedures (2002-2011) were systematically searched and re-
viewed. End points were periprocedural mortality, stroke, and spinal cord ischemia.

Results: A total of 50 studies including 1886 patients were included. Perioperative mortality ranged from $1.6 \%$ to $25.0 \%$ with a pooled event ratio of $10.8 \%(95 \%$ confidence intervals $[\mathrm{CI}], 9.3-12.5)$. Perioperative stroke, regardless of severity, ranged from $0.8 \%$ to $25.0 \%$ (pooled ratio $6.9 \%$; $95 \%$ CI, $5.7 \%-8.4$ ), and spinal cord ischemia, including permanent and transitory events, ranged from $1.0 \%$ to $25.0 \%$ (pooled ratio, $6.8 \%$; $95 \%$ CI, 5.6-8.2). Neurologic but no mortality risk was affected by timing and center volume with decreased rates in more recent and higher volume studies. In dissected aorta, perioperative mortality rate was 9.8\% (95\% CI, 7.7-12.4), stroke 4.3\% (95\% CI, 3.0-6.3), and spinal cord ischemia 5.8\% (95\% CI, 4.2-7.9). Perioperative mortality was higher in diseases that extended to the ascending aorta $(15.1 \%$ vs $7.6 \%$; odds ratio, $2.8 ; 95 \% \mathrm{CI}, 1.17-6.7 ; P=.021)$, whereas there were no significant differences in the neurologic risks of stroke or spinal cord ischemia.

Conclusions: Hybrid repair of the aortic arch carries not negligible risks of perioperative mortality and neurologic morbidity. Risk of neurologic complications has decreased with timing and center volume and may be limited in dissection repairs. However, contemporary information on aortic hybrid arch procedures is mainly provided by small case series or retrospective studies with wide range of results. (J Thorac Cardiovasc Surg 2012;144:1286-1300)

丹 Supplemental material is available online.

Repair of aortic arch diseases remains a surgical challenge. Despite significant advances in operative care, conventional treatment is still associated with significant incidence of perioperative mortality and neurologic complications. Furthermore, many patients are denied surgical intervention owing to their significant comorbidities.

From Vascular Surgery, ${ }^{\mathrm{a}}$ Ospedale San Camillo, Rome, Italy; Vascular Surgery, ${ }^{\mathrm{b}}$ Ospedale S. Maria della Misericordia, Perugia, Italy; Cardiovascular Surgery, ${ }^{\text {c }}$ Basel, Switzerland; Cardiology, ${ }^{\mathrm{d}}$ Hospital Val d'Hebron Barcelona, Barcelona, Spain; Cardiovascular Radiology, ${ }^{\text {e }}$ San Salvatore Hospital, Pesaro, Italy; Interventional Cardiology, ${ }^{\mathrm{f}}$ University of Rostock, Rostock, Germany; Radiology, ${ }^{\mathrm{g}}$ Toulouse, France; and Cardiovascular Surgery, ${ }^{\text {h }}$ Brugge, Belgium.

Literature search was in part supported by an Educational Grant from Medtronic, Inc.

There was no involvement of the sponsor in interpretation of data and drafting the article. All authors were independent participants and did not receive financial compensation for their involvement in this article.

Disclosures: Authors have nothing to disclose with regard to commercial support.

Received for publication March 29, 2012; revisions received May 20, 2012; accepted

for publication June 8, 2012; available ahead of print July 12, 2012.

Address for reprints: Paola De Rango, MD, Unit of Vascular and Endovascular Sur-

gery, Hospital S.M. Misericordia, Loc. S. Andrea delle Fratte, 06134 Perugia, Italy

(E-mail: plderango@gmail.com or pderango@unipg.it).

$0022-5223 / \$ 36.00$

Copyright (c) 2012 by The American Association for Thoracic Surgery

http://dx.doi.org/10.1016/j.jtcvs.2012.06.013
Recent advances in thoracic stent grafts have enhanced the management of thoracic aorta disorders. Nevertheless, when the disease extends into the aortic arch, conventional endovascular techniques are challenged.

Endovascular exclusion of the aortic arch combined with an open surgical component, effectively named "hybrid" aortic arch repair, has been recently introduced as an effective alternative to decrease morbidity and mortality in selected patients. ${ }^{1,2}$ However, the available data on clinical outcomes are limited, particularly for patients with dissection. In addition, several different approaches to hybrid arch repair have been described.

The aim of this study was to perform a systematic review of the literature on hybrid operative strategies for aortic arch repair with a pooled analysis of periprocedural clinical outcomes (mortality and neurologic complications) particularly when applied for aortic dissection.

\section{METHODS \\ Search Strategy}

A literature search was undertaken to identify all published studies in the past 10 years reporting on hybrid treatment of aortic arch diseases. Candidate studies in English were sought through a computerized search of the Ovid and MEDLINE (National Library of Medicine, Bethesda, Md) databases for the period of January 2002 to November 2011. Key words entered in this search were "hybrid" or "debranching," with "aortic arch," "endovascular," and "dissection." Additionally, manual evaluation of the reference lists of the retrieved articles and reviews on this area subject was undertaken. 


\section{Abbreviations and Acronyms \\ $95 \% \mathrm{CI}=95 \%$ confidence interval \\ $\mathrm{OR}=$ odds ratio}

\section{Study Selection}

Studies were considered for inclusion on the basis of these criteria:

- Reporting on combined open surgical and endovascular repair of aortic arch

- Including at least 5 patients treated with this method

- Reporting on clinical outcome

In some articles, patients treated with hybrid procedures were not the total but a subpopulation and no detailed information was given for this subgroup. Data were included if there was a separate description of these patients or if relevant data could be sufficiently retrieved from the article.

Studies containing duplicate data were excluded and the ones with the most recent or the best-documented material from the same authors were used for analysis.

An extensive effort was made to individualize outcomes of patients with aortic dissection. However, many articles combined outcomes for arch aneurysm and dissection and did not report results separately for the dissection cohort. Furthermore, acute, subacute, and chronic dissections, as well as type A and B dissections, were usually combined. Data on dissected aorta were separately analyzed only when clear information was provided for this subgroup.

\section{Definitions}

Inasmuch as the variety of hybrid techniques is strictly related to the availability of landing zones and disease extension, 3 subgroups of hybrid repairs were separately analyzed: "arch debranching," "frozen elephant trunk," and "stented elephant trunk with endovascular repair of thoracoabdominal aorta.",

Aortic arch zones were classified according to Mitchell/Ishimaru: zone 0 is proximal to the innominate artery, zone 1 involves the origin of the left common carotid artery, zone 2 includes the left subclavian artery, zone 3 extends beyond the origin of the left subclavian artery to the beginning of descending thoracic aorta, and zone 4 involves the descending thoracic aorta. $^{3}$

\section{Statistical Analysis}

Data retrieved for each study were entered in a purposed designed dataset, pooled, and analyzed according to published guidelines for synthesis of information from the existing literature (Moose). Study quality was rated according to formal assessment check list. ${ }^{4}$ Meta-analysis was conducted using a specific statistical package (Comprehensive Meta-analysis Package, version 2; Biostat, Englewood, NJ). Primary outcome measures were perioperative (30-day or in-hospital) incidence of neurologic complications (stroke and spinal cord ischemia) and mortality. Event rates, ranges, and corresponding $95 \%$ confidence intervals $(95 \% \mathrm{CI})$ were used to summarize data pooled from all eligible published studies.

A separate analysis of the outcome measures was performed in 3 subgroups by type of hybrid repair (debranching, frozen elephant trunk, stented elephant trunk) and in the subgroup of repairs for dissections when results were clearly given in this type of aortic disease. To evaluate whether results were affected by time experience or volume center, we repeated analyses with data breakdown by publication year (studies before 2007 and since 2007 ) and by center volume (studies with $\geq 20$ and $<20$ volume).
Secondary analyses were performed in studies by comparing zone 0 versus zone 1 .

The differences in proportions of complication rates by zone were analyzed using the $\chi^{2}$ and Fisher exact tests. The estimates of outcome rates, the corresponding odds ratios (OR), and the differences between event rates were given with the $95 \% \mathrm{CI}$.

\section{RESULTS}

\section{Search Results}

The literature search (see Figure 1) identified 153 potential publications. Seventy-four were considered relevant for the purposes of this review. Sixteen were duplicate publications without details on outcome and were excluded. Six were excluded because they were reviews of other studies, and 2 were excluded because they did not provide detailed results, leaving a total of 50 studies available for the analysis including 1886 patients (complete list of included and excluded studies shown as E-References online only).

Main characteristics of the included studies are shown in Tables 1 to $4 .^{2,5-\mathrm{E} 53}$ Most were retrospective studies, and there was no level 1 evidence. In most studies, different types of aortic disease and hybrid repair were combined. Furthermore, most series provided outcomes for emergency and elective repair without providing separate outcomes. Finally, some studies were mainly focused on high-risk patients (unfit for open surgery), whereas highrisk and average risk patients were combined in other studies and the issue was not always clarified to detect separate information.

Twenty-seven studies including 642 patients focused on arch debranching., ${ }^{2,13-15,20-E 42}$

Seventeen studies and 1103 patients were available for frozen elephant trunk technique. ${ }^{5-12,16-19, E 43-E 47}$ Nine studies and 141 patients were available for stented elephant trunk. ${ }^{15, \mathrm{E} 34, \mathrm{E} 35, \mathrm{E} 48-\mathrm{E} 53}$

When information on different subgroup techniques (debranching, frozen elephant trunk, or stented elephant trunk) was provided by the same study, data were separately extracted and analyzed for each specific subgroup. In 3 studies, information was available for both debranching and stented elephant trunk techniques. ${ }^{15, \mathrm{E} 34, \mathrm{E} 35}$ In another study, different outcomes were provided in different population settings receiving frozen elephant trunk and these were separately analyzed. ${ }^{12}$

Data from 17 studies were available for analysis of patients with acute/chronic type $\mathrm{A}$ and $\mathrm{B}$ aortic dissection., ${ }^{2,5-20}$

Details by landing zone were available from 19 studies. $^{2,13,14, \mathrm{E} 21-\mathrm{E} 28, \mathrm{E} 30, \mathrm{E} 31, \mathrm{E} 33, \mathrm{E} 34, \mathrm{E} 36, \mathrm{E} 37, \mathrm{E} 41, \mathrm{E} 53}$

\section{Outcomes}

Primary outcome measures in the individual studies and pooled data are shown in Figures 2, 3, and 4. 


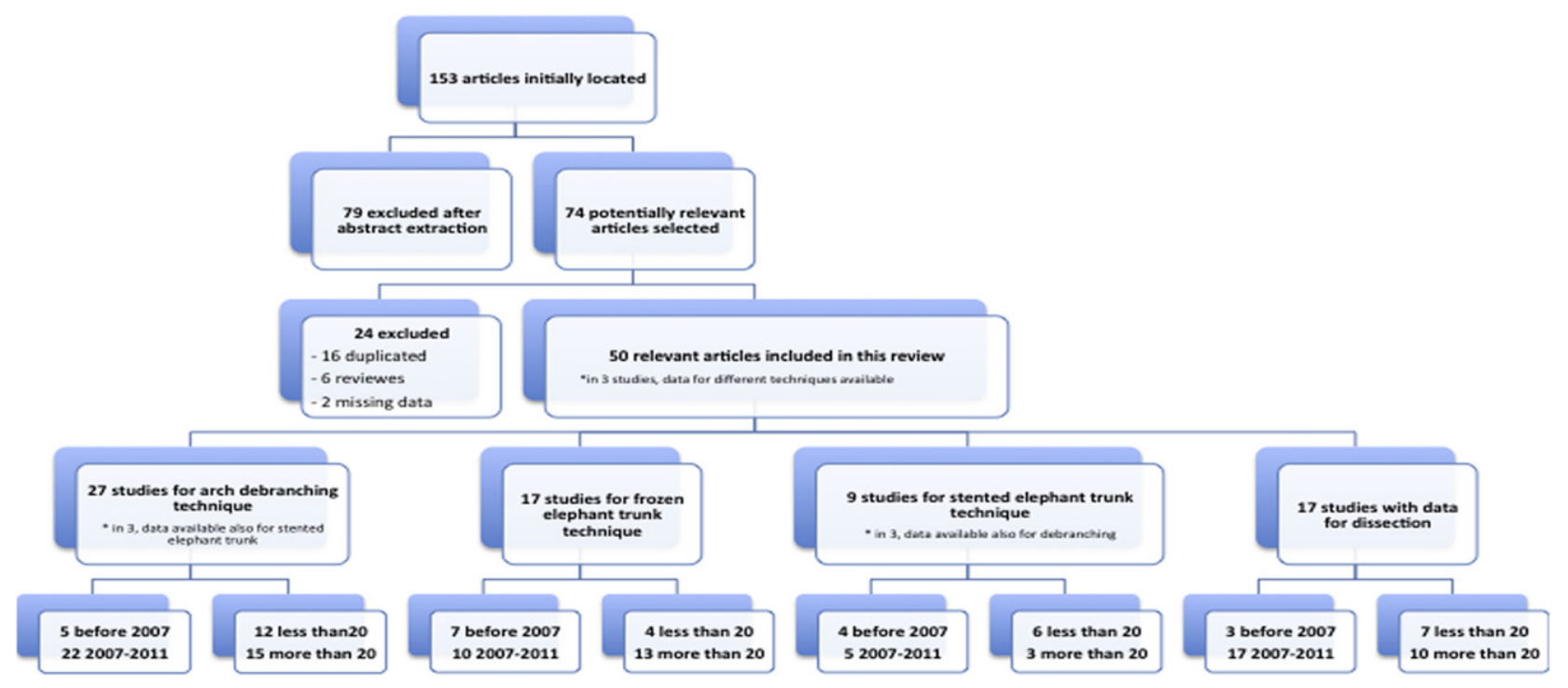

FIGURE 1. Search strategy.

Mortality (Figure 2). Perioperative mortality ranged from $1.6 \%$ to $25.0 \%$ with a pooled event ratio of $10.8 \%$ (95\% CI, 9.3-12.5). In the group of 27 studies focused on debranching, mortality ranged from $2.0 \%$ to $23.7 \%$ with a pooled event ratio of $11.9 \% \quad(95 \% \quad \mathrm{CI}$, 9.4-15.1) 2,13-15,20-E42 (Figure 2, A).

In the 17 studies reporting on frozen elephant trunk, perioperative mortality ranged from $1.7 \%$ to $18.2 \%$ with a pooled event ratio of $9.8 \%(95 \% \mathrm{CI}$, 8.0-11.9) 5-12,16-19,E43-E47 (Figure 2, B).

In the 9 studies reporting on stented elephant trunk, perioperative mortality ranged from $1.6 \%$ to $25.0 \%$ with a pooled event ratio of $13.2 \% \quad(95 \%$ CI, 7.8-21.4) ${ }^{15, \mathrm{E} 34, \mathrm{E} 35, \mathrm{E} 48-\mathrm{E} 53}$ (Figure 2, C).

Neurologic outcomes (Figures 3 and 4). Perioperative stroke, regardless of severity, ranged from $0.8 \%$ to $25.0 \%$ with a pooled event ratio of $6.9 \%(95 \% \mathrm{CI}$, 5.7-8.4). Stroke rates ranged from $0.8 \%$ to $18.8 \%$ in the debranching group $(7.3 \% ; 95 \% \mathrm{CI}, 5.3-10)$, from $1.0 \%$ to $16.0 \%$ in the frozen elephant trunk group $(6.2 \% ; 95 \%$ CI, $4.8-8.0$ ), and from $1.6 \%$ to $25.0 \%$ in the stented elephant trunk group $(10.9 \% ; 95 \%$ CI, 5.8-19.7) (Figure 3, A-C).

TABLE 1. Dissection

\begin{tabular}{|c|c|c|c|c|c|c|c|c|c|}
\hline \multirow[b]{2}{*}{ First author } & \multirow[b]{2}{*}{ Year } & \multirow[b]{2}{*}{ Dissection (N) } & \multirow[b]{2}{*}{ Total (N) } & \multirow[b]{2}{*}{$\mathbf{A}$} & \multirow[b]{2}{*}{ B } & \multirow[b]{2}{*}{ Acute } & \multicolumn{3}{|c|}{ Thirty days or in hospital } \\
\hline & & & & & & & Death & Stroke & Spinal ischemia \\
\hline Baraki $^{5}$ & 2007 & 21 & 39 & 15 & 6 & 7 & 3 & $?$ & 0 \\
\hline Chavan $^{6}$ & 2005 & 15 & 22 & 11 & 4 & 1 & 1 & $?$ & $?$ \\
\hline Chen $^{7}$ & 2010 & 28 & 28 & 28 & 0 & 27 & 4 & 3 & 3 \\
\hline Di Eusanio ${ }^{8}$ & 2011 & 49 & 49 & 39 & 10 & 0 & 5 & 0 & 6 \\
\hline Fleck $^{9}$ & 2002 & 8 & 8 & 8 & 0 & ? & 1 & 1 & 0 \\
\hline Gorlitzer $^{10}$ & 2007 & 5 & 7 & 4 & 1 & 3 & 0 & 1 ? & 0 \\
\hline Ingrund $^{2}$ & 2010 & 9 & 12 & 3 & 6 & 9 & 1 & 0 & 0 \\
\hline Jakob $1^{11}$ & 2008 & 22 & 22 & 22 & 0 & 22 & 2 & 2 & 0 \\
\hline Jakob $2^{12}$ & 2011 & 102 & 102 & 102 & 0 & 0 & 13 & 3 & 8 \\
\hline Jakob $3^{12}$ & 2011 & 88 & 88 & 88 & 0 & 88 & 16 & 5 & 5 \\
\hline $\mathrm{Lu}^{13}$ & 2011 & 18 & 22 & 0 & 18 & 5 & 2 & 1 & 0 \\
\hline $\mathrm{Ma}^{14}$ & 2011 & 24 & 24 & $?$ & $?$ & $?$ & 0 & 0 & 0 \\
\hline Obitsu $^{15}$ & 2011 & 9 & 17 & 9 & 0 & 0 & 0 & 0 & 0 \\
\hline Shimamura $1^{16}$ & 2008 & 57 & 126 & 41 & 16 & 31 & 2 & $?$ & 4 \\
\hline Shimamura $2^{17}$ & 2009 & 33 & 69 & 13 & 20 & 12 & ? & 1 & $?$ \\
\hline $\operatorname{Sun}^{18}$ & 2011 & 291 & 291 & 291 & 0 & 148 & 9 & 7 & 7 \\
\hline Uchida $^{19}$ & 2006 & 35 & 35 & 35 & 0 & 35 & 2 & 0 & 0 \\
\hline Wang $^{20}$ & 2008 & 14 & 20 & 0 & 14 & ? & 1 & 1 & 0 \\
\hline
\end{tabular}


TABLE 2. Debranching (group I)

\begin{tabular}{|c|c|c|c|c|c|c|c|c|c|c|c|c|c|}
\hline First author & High risk & Emer. & $\mathbf{R} / \mathbf{P}$ & Year & Years & Total & Debranching & $\mathbf{M} / \mathbf{F}$ & Age & Dissection & Stage $1 / 2$ & $\begin{array}{c}\text { Partial } \\
\text { or Total }\end{array}$ & $\begin{array}{l}\text { Zones } \\
0,1,2\end{array}$ \\
\hline Bergeron $^{\mathrm{E} 21}$ & Yes, $48 \%$ & Yes, $15 \%$ & $\mathrm{P}$ & 2006 & 2001-2005 & 29 & 29 & $23 / 6$ & NR & 13 & $0 / 29$ & $16 / 11$ & $15,10,4$ \\
\hline Canaud $^{\mathrm{E} 22}$ & Yes, 44 & Yes, $27 \%$ & $\mathrm{R}$ & 2010 & $1998-2008$ & 44 & 10 & NR & NR & NR & NR & $4 / 6$ & $6,4,24$ \\
\hline Carrel $^{\mathrm{E} 23}$ & Yes, 6 & $?$ & $\mathrm{R}$ & 2005 & $2001-2003$ & 6 & 6 & NR & NR & 0 & NR & $1 / 5$ & $5,1,0$ \\
\hline Chan $^{\mathrm{E} 24}$ & Yes, 16 & Yes, 7 ? & $\mathrm{R}$ & 2008 & $2005-2007$ & 16 & 16 & $13 / 3$ & 65 & 6 & $16 / 0$ & $9 / 5$ & $5,8,3$ \\
\hline Chen $^{\mathrm{E} 25}$ & No & Yes & $\mathrm{R}$ & 2008 & $2005-2008$ & 6 & 6 & NR & NR & 1 & $6 / 0$ & $0 / 6$ & $6,0,0$ \\
\hline Chiesa ${ }^{\mathrm{E} 26}$ & No & No? & $\mathrm{R}$ & 2010 & 1999-2009 & 116 & 51 & $43 / 43$ & NR & 21 & NR & $27 / 24$ & $24,27,65$ \\
\hline Ferrero $^{\text {E27 }}$ & Yes, $40 \%$ & Yes & $\mathrm{R}$ & 2011 & $2005-2010$ & 27 & 27 & $22 / 5$ & 71.6 & 4 & NR & $16 / 11$ & $11,4,12$ \\
\hline Geisbüsch $^{\mathrm{E} 28}$ & Yes, $47 \%$ & Yes, $34 \%$ & $\mathrm{R}$ & 2011 & $1997-2009$ & 47 & 47 & $33 / 14$ & 64 & 15 & $50 \% ?$ & $32 / 15$ & $10,25,12$ \\
\hline Gelpi $^{\mathrm{E} 29}$ & Yes, 15 & No & $\mathrm{R}$ & 2010 & 2004-2009 & 15 & 15 & $12 / 3$ & 70 & 2 & $0 / 15$ & NR & NR \\
\hline Gottardi $^{\mathrm{E} 30}$ & Yes & No? & $\mathrm{R}$ & 2008 & $1996-2007$ & 73 & 60 & $34 / 15$ & NR & 9 & $36 / 24$ & $60 / 0$ & $0,36,24$ \\
\hline Weigang $^{\text {E31 }}$ & Yes, 26 & No & $\mathrm{R}$ & 2009 & NR & 26 & 26 & $20 / 6$ & NR & 6 & 1 st. prefer. & $0 / 26$ & $26,0,0$ \\
\hline $\mathrm{Ham}^{\mathrm{E} 32}$ & Yes & Yes & $\mathrm{R}$ & 2011 & $2005-2011$ & NR & 16 & NR & NR & NR & NR & NR & NR \\
\hline Holt $^{\mathrm{E} 33}$ & No & Yes & $\mathrm{R}$ & 2010 & 2001-2009 & 78 & 39 & NR & NR & NR & NR & $16 / 23$ & $9,17,13$ \\
\hline Hughes $1^{\mathrm{E} 34}$ & No & Yes & $\mathrm{R}$ & 2009 & $2005-2008$ & 21 & 21 & NR & NR & NR & $21 / 0$ & $0 / 21$ & $12,9,0$ \\
\hline Ingrund $^{2}$ & No & Yes, 12 & $\mathrm{R}$ & 2010 & 2007-2009 & 12 & 12 & $6 / 6$ & 54 & 9 & NR & $8 / 4$ & $4,8,0$ \\
\hline $\mathrm{Lee}^{\mathrm{E} 35}$ & Yes, $86 \%$ & Yes, $5.4 \%$ & $\mathrm{R}$ & 2011 & $2005-2009$ & 37 & 37 & $23 / 14$ & 63 & 13 & $0 / 37$ & NR & NR \\
\hline $\mathrm{Lu}^{13}$ & No & Yes, $22 \%$ & $\mathrm{R}$ & 2011 & 2001-2009 & 22 & 18 & $19 / 3$ & 49 & 18 & $3 / 15$ & NR & $1,16,11$ \\
\hline $\mathrm{Ma}^{14}$ & No & $?$ & $\mathrm{R}$ & 2011 & $2005-2010$ & 24 & 24 & $16 / 8$ & 42 & 24 & NR & NR & $3,10,11$ \\
\hline Murashita $^{\mathrm{E} 36}$ & Yes & No & $\mathrm{R}$ & 2011 & $2007-2010$ & 27 & 27 & $22 / 5$ & 76 & NR & NR & $23 / 4$ & $4,19,4$ \\
\hline Obitsu $^{15}$ & No & No & $\mathrm{R}$ & 2011 & $1995-2008$ & 25 & 25 & NR & NR & 0 & $22 / 3$ & $20 / 5$ & NR \\
\hline Saleh $^{\mathrm{E} 37}$ & Yes, 16 & No & $\mathrm{R}$ & 2007 & $2005-2007$ & 16 & 16 & $12 / 4$ & 67 & 0 & $0 / 16$ & $0 / 16$ & $16,0,0$ \\
\hline Schumacher ${ }^{\mathrm{E} 38}$ & Yes, 25 & Yes, $32 \%$ & $\mathrm{R}$ & 2006 & $1998-2006$ & 25 & 25 & $20 / 5$ & 65 & 2 & $9 / 16$ & $16 / 9$ & $9,16,0$ \\
\hline Sueda ${ }^{\text {E39 }}$ & Yes & No & $\mathrm{R}$ & 2003 & NR & 7 & 7 & NR & NR & 0 & NR & $5 / 2$ & NR \\
\hline Szeto ${ }^{\mathrm{E} 40}$ & Yes, 8 & Yes, $2.5 \%$ & $\mathrm{R}$ & 2007 & $2005-$ & 8 & 8 & $7 / 1$ & 67 & 1 & $8 / 0$ & $0 / 8$ & NR \\
\hline Vallejo $^{\mathrm{E} 41}$ & Yes & Yes & $\mathrm{R}$ & 2011 & $2002-2011$ & 38 & 38 & $27 / 11$ & 65.4 & 20 & $24 / 14$ & $11 / 27$ & $20,12,8$ \\
\hline Wang $^{20}$ & Yes, 20 & Yes & $\mathrm{R}$ & 2008 & $2002-2007$ & 34 & 20 & NR & NR & 14 & $15 / 5$ & NR & $4,11,5$ \\
\hline Zhou $^{\text {E42 }}$ & Yes, 31 & No & $\mathrm{R}$ & 2006 & 2004-2005 & 31 & 16 & NR & NR & 0 & $8 / 8$ & NR & $11,5,0$ \\
\hline
\end{tabular}

Emer, Emergencies; $R$, retrospective; $P$, prospective; $N R$, not registered.

Perioperative spinal cord ischemia including permanent and transitory events ranged from $1.0 \%$ to $25.0 \%$ with a pooled event ratio of $6.8 \%(95 \% \mathrm{CI}, 5.6-8.2)$. Rates ranged from $1.0 \%$ to $14.3 \%$ in the debranching group $(4.3 \%$; $95 \%$ CI, $2.8-6.4)$, from $1.3 \%$ to $25.0 \%$ in the frozen elephant trunk group $(7.9 \% ; 95 \% \mathrm{CI}, 6.3-9.9)$, and from

TABLE 3. Characteristics in frozen elephant trunk (group II)

\begin{tabular}{|c|c|c|c|c|c|c|c|c|c|c|}
\hline First author & Year & Study years & Total (N) & FELT (N) & Age & $\mathbf{M} / \mathbf{F}$ & $\mathbf{R} / \mathbf{P}$ & High risk & Emergency & Dissection \\
\hline Baraki $^{5}$ & 2007 & 2001-2006 & 39 & 39 & $62 \mathrm{y}$ & M 24/F 15 & $\mathrm{R}$ & No & No & 21 \\
\hline Chavan $^{6}$ & 2005 & $?$ & 22 & 22 & $64 \mathrm{y}$ & M 13/F 9 & $\mathrm{P}$ & No & No & 15 \\
\hline Chen $^{7}$ & 2010 & 2004-2009 & 28 & 28 & $51.2 \mathrm{y}$ & M 22/F 6 & $\mathrm{R}$ & No & Yes, n 27 & 28 \\
\hline Fleck $^{9}$ & 2002 & 2001-2002 & 8 & 8 & $55 \mathrm{y}$ & M 5/F 3 & $\mathrm{R}$ & Emerg? & Yes & 8 \\
\hline Flores $^{\mathrm{E} 43}$ & 2006 & 1996-2004 & 25 & 25 & $73 y$ & M 19/F 6 & $\mathrm{R}$ & No & $?$ & 5 \\
\hline Di Eusanio $^{8}$ & 2011 & $2007-2010$ & 49 & 49 & $59 \mathrm{y}$ & M 43/F 6 & $\mathrm{R}$ & No & No & 49 \\
\hline Gorlitzer $^{10}$ & 2007 & $2005-2006$ & 7 & 7 & $62 \mathrm{y}$ & $\mathrm{M} 5 / \mathrm{F} 2$ & $\mathrm{R}$ & No & Yes & 5 \\
\hline Jakob $1^{11}$ & 2008 & 2001-2007 & 22 & 22 & $57 \mathrm{y}$ & M 17/F 5 & $\mathrm{R}$ & No & Yes & 22 \\
\hline Jakob $2^{12}$ & 2011 & $2005-2010$ & 102 & 102 & $57 \mathrm{y}$ & M 82/F 20 & $P$ register & No & No & 102 \\
\hline Jakob $3^{12}$ & 2011 & $2005-2010$ & 88 & 88 & $59 \mathrm{y}$ & M 68/F 20 & $P$ register & No & Yes, n 88 & 88 \\
\hline Jakob $4^{12}$ & 2011 & $2005-2010$ & 84 & 84 & $66 \mathrm{y}$ & M 54/F 20 & $\mathrm{P}$ register & No & No & 84 \\
\hline Miyairi $^{\mathrm{E} 44}$ & 2002 & $1996-2000$ & 19 & 19 & $69 \mathrm{y}$ & M 15/F 4 & $\mathrm{R}$ & No & No & 2 \\
\hline Mizuno $^{\text {E45 }}$ & 2003 & 1996-2000 & 8 & 8 & $75 \mathrm{y}$ & $\mathrm{M} 6 / \mathrm{F} 2$ & $\mathrm{R}$ & No & No & 0 \\
\hline Sakurai $^{\text {E46 }}$ & 2006 & 1997-2001 & 23 & 23 & $70 \mathrm{y}$ & M 21/F 2 & $\mathrm{R}$ & Yes & No & 1 \\
\hline Shimamura $1^{16}$ & 2008 & 1994-2004 & 126 & 126 & $67.8 \mathrm{y}$ & M 86/F 40 & $\mathrm{R}$ & No & Yes, n 37 & 57 \\
\hline Shimamura $2^{17}$ & 2009 & 2004-2007 & 69 & 69 & $66.2 \mathrm{y}$ & M 55/F 14 & $\mathrm{R}$ & No & Yes, n 13 & 33 \\
\hline $\operatorname{Sun}^{18}$ & 2011 & $2003-2008$ & 291 & 291 & $45 \mathrm{y}$ & M 238/F 53 & $\mathrm{R}$ & No & Yes, n 148 & 291 \\
\hline Uchida $1^{\mathrm{E} 47}$ & 2010 & 1997-2008 & 58 & 58 & $73.9 \mathrm{y}$ & M 52/F 6 & $\mathrm{R}$ & Yes & $?$ & 0 \\
\hline Uchida $2^{19}$ & 2006 & 1997-2002 & 35 & 35 & $67.8 \mathrm{y}$ & M 13/F 22 & $\mathrm{R}$ & No & Yes, n 35 & 35 \\
\hline
\end{tabular}

FELT, Frozen elephant trunk; $R$, retrospective; $P$, prospective; Emerg, emergency. 
TABLE 4. Characteristics in stented elephant trunk (group III)

\begin{tabular}{|c|c|c|c|c|c|c|c|c|c|c|c|}
\hline First author & Year & Study years & $\begin{array}{l}\text { Total } \\
(\mathbf{N})\end{array}$ & $\begin{array}{l}\text { ET } \\
(\mathbf{N})\end{array}$ & Age & $\mathbf{M} / \mathbf{F}$ & $\mathbf{R} / \mathbf{P}$ & High risk & Dissection & Emerg & Zone 0, 1, 2 \\
\hline Carroccio $^{\mathrm{E} 48}$ & 2005 & $6 y$ & 12 & 12 & 69 & M 7/F 5 & $\mathrm{R}$ & Yes, n 12 & 0 & Yes $16 \%$ & \\
\hline Greenberg $^{\mathrm{E} 49}$ & 2005 & $2000-2004$ & 22 & 22 & 73 & M 11/F 11 & $\mathrm{R}$ & Yes & 4 & Yes $4.5 \%$ & \\
\hline Hughes $^{\text {E34 }}$ & 2009 & 2005-2008 & 7 & 7 & $?$ & $?$ & $\mathrm{R}$ & No & $?$ & Yes & $0=3,1=?, 2=4$ \\
\hline Kawaharada ${ }^{\mathrm{E} 50}$ & 2009 & $2001-2007$ & 31 & 31 & 70 & M 24/F 7 & $\mathrm{R}$ & No & 4 & No & \\
\hline Kieffer $^{\mathrm{E} 51}$ & 2005 & $1995-2003$ & 16 & 16 & 73 & M 14/F 2 & $\mathrm{R}$ & No & 0 & No & $0=10,1=6,2=0$ \\
\hline $\operatorname{Jim}^{\mathrm{E} 52}$ & 2011 & 2005-2009 & 10 & 10 & 67,5 & M 3/F 7 & $\mathrm{R}$ & Yes, n 10 & 3 & No & \\
\hline $\mathrm{Lee}^{\mathrm{E} 35}$ & 2011 & $2005-2009$ & 58 & 21 & 68 & M 13/F 8 & $\mathrm{R}$ & Yes, $86 \%$ & 4 & No & \\
\hline Obitsu $^{15}$ & 2011 & $1995-2008$ & 17 & 17 & $?$ & $?$ & $\mathrm{R}$ & No & 9 & No & \\
\hline Tse $\mathrm{E}^{\mathrm{E} 3}$ & 2004 & $1999-2003$ & 5 & 5 & $?$ & $?$ & $\mathrm{R}$ & No & $?$ & Yes & $0=0,1=3,2=2$ \\
\hline
\end{tabular}

$R$, Retrospective; $P$, prospective; $E T$, elephant trunk; Emerg, emergency.

$1.6 \%$ to $14.3 \%$ in the stented elephant trunk group $(7.2 \%$; 95\% CI, 3.5-14.5) (Figure 4, A-C).

Dissections (Figure 5, $\boldsymbol{A}-\boldsymbol{C}$ ). For 828 patients operated on for aortic dissection (including types $\mathrm{A}$ and $\mathrm{B}$, chronic, and acute), data on outcomes were available..$^{2,5-20}$
Characteristics of patients with dissections are shown in Table 1. In patients with aortic dissection, perioperative mortality from hybrid repair ranged from $0.0 \%$ to $14.3 \%$ with a pooled event ratio of $9.8 \%$ (95\% CI, 7.7-12.4). Regarding periprocedural neurologic complications, stroke ranged from $0.0 \%$ to $12.5 \%$ with a pooled event ratio of

\section{Perioperative mortality in debranching}

\begin{tabular}{|c|c|c|c|c|c|c|c|c|c|c|}
\hline \multirow[t]{2}{*}{$\underline{\text { Study name }}$} & \multirow[t]{2}{*}{ Mortal ity periop } & \multicolumn{5}{|c|}{$\underline{\text { Statistics for each study }}$} & & \multicolumn{2}{|c|}{ Event rate and $95 \% \mathrm{Cl}$} & \\
\hline & & $\begin{array}{l}\text { Event } \\
\text { rate }\end{array}$ & $\begin{array}{c}\text { Lower } \\
\text { limit }\end{array}$ & $\begin{array}{l}\text { Upper } \\
\text { limit }\end{array}$ & Z-Value & p-Value & & & & \\
\hline Bergeron 2006 & debranching & 0,069 & 0,017 & 0,238 & $-3,552$ & 0,000 & & 1 & 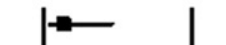 & \\
\hline Canaud 2010 & debranching & 0,200 & 0,050 & 0,541 & $-1,754$ & 0,080 & & & & \\
\hline Carrel 2005 & debranching & 0,071 & 0,004 & 0,577 & $-1,748$ & 0,081 & & & & \\
\hline Chan 2008 & debranching & 0,029 & 0,002 & 0,336 & $-2,436$ & 0,015 & & & & \\
\hline Chen.12008 & debranching & 0,071 & 0,004 & 0,577 & $-1,748$ & 0,081 & & & & \\
\hline Chiesa 2010 & debranching & 0,059 & 0,019 & 0,167 & $-4,659$ & 0,000 & & & & \\
\hline Ferrero 2011 & debranching & 0,111 & 0,036 & 0,293 & $-3,396$ & 0,001 & & & & \\
\hline Geisbusch 2011 & debranching & 0,191 & 0,103 & 0,329 & $-3,885$ & 0,000 & & & & \\
\hline Gelpi 2010 & debranching & 0,067 & 0,009 & 0,352 & $-2,550$ & 0,011 & & & & \\
\hline Gottardi 2008 & debranching & 0,033 & 0,008 & 0,124 & $-4,682$ & 0,000 & & & & \\
\hline Ham 2011 & debranching & 0,063 & 0,009 & 0,335 & $-2,622$ & 0,009 & & & & \\
\hline Holt 2010 & debranching & 0,026 & 0,004 & 0,161 & $-3,591$ & 0,000 & & & & \\
\hline Hughes.12009 & debranching & 0,023 & 0,001 & 0,277 & $-2,629$ & 0,009 & & & & \\
\hline Ingrund 2010 & debranching & 0,167 & 0,042 & 0,477 & $-2,078$ & 0,038 & & & & \\
\hline Lee.1 2011 & debranching & 0,162 & 0,075 & 0,317 & $-3,682$ & 0,000 & & & & \\
\hline Lu 2011 & debranching & 0,111 & 0,028 & 0,352 & $-2,773$ & 0,006 & & & & \\
\hline Ma 2011 & debranching & 0,020 & 0,001 & 0,251 & $-2,724$ & 0,006 & & & & \\
\hline Murashita 2011 & debranching & 0,037 & 0,005 & 0,221 & $-3,197$ & 0,001 & & & & \\
\hline Obitsu.1 2011 & debranching & 0,040 & 0,006 & 0,235 & $-3,114$ & 0,002 & & & & \\
\hline Saleh 2007 & debranching & 0,063 & 0,009 & 0,335 & $-2,622$ & 0,009 & & & & \\
\hline Schumacher 2006 & debranching & 0,200 & 0,086 & 0,400 & $-2,773$ & 0,006 & & & & \\
\hline Sueda 2003 & debranching & 0,143 & 0,020 & 0,581 & $-1,659$ & 0,097 & & & & \\
\hline Szeto 2007 & debranching & 0,125 & 0,017 & 0,537 & $-1,820$ & 0,069 & & & & \\
\hline Vallejo 2011 & debranching & 0,237 & 0,128 & 0,396 & $-3,066$ & 0,002 & & & & \\
\hline Wang 2008 & debranching & 0,050 & 0,007 & 0,282 & $-2,870$ & 0,004 & & & & \\
\hline Weigang 2009 & debranching & 0,154 & 0,059 & 0,345 & $-3,136$ & 0,002 & & & & \\
\hline \multirow[t]{3}{*}{ Zhou 2006} & debranching & 0,063 & 0,009 & 0,335 & $-2,622$ & 0,009 & & & & \\
\hline & & 0,119 & 0,094 & 0,151 & $-14,489$ & 0,000 & & & $\bullet$ & \\
\hline & & & & & & & $-1,00$ & $-0,50$ & 0,00 & 1,00 \\
\hline
\end{tabular}

A

FIGURE 2. Perioperative mortality in hybrid arch repair. A, Debranching technique. B, Frozen elephant trunk technique. C, Stented elephant trunk technique. $C I$, Confidence interval; ET, elephant trunk. 
Perioperative mortality in frozen elephant trunk

\begin{tabular}{|c|c|c|c|c|c|c|c|c|c|}
\hline \multirow[t]{2}{*}{ Study name } & \multirow[t]{2}{*}{ Mortality periop } & \multicolumn{5}{|c|}{ Statistics for each study } & & \multicolumn{2}{|c|}{ Event rate and $95 \% \mathrm{Cl}$} \\
\hline & & $\begin{array}{l}\text { Event } \\
\text { rate }\end{array}$ & $\begin{array}{c}\text { Lower } \\
\text { limit }\end{array}$ & $\begin{array}{l}\text { Upper } \\
\text { limit }\end{array}$ & Z-Value & p-Value & & & \\
\hline Baraki 2007 & Frozen ET & 0,128 & 0,054 & 0,273 & $-4,002$ & 0,000 & & & \\
\hline Chavan 2005 & Frozen ET & 0,045 & 0,006 & 0,261 & $-2,975$ & 0,003 & & & \\
\hline Chen.2 2010 & Frozen ET & 0,143 & 0,055 & 0,324 & $-3,318$ & 0,001 & & & \\
\hline Di Eusanio 2011 & Frozen ET & 0,102 & 0,043 & 0,223 & $-4,608$ & 0,000 & & & \\
\hline Fleck 2002 & Frozen ET & 0,125 & 0,017 & 0,537 & $-1,820$ & 0,069 & & & \\
\hline Flores 2006 & Frozen ET & 0,120 & 0,039 & 0,313 & $-3,237$ & 0,001 & & & \\
\hline Gorlitzer 2007 & Frozen ET & 0,063 & 0,004 & 0,539 & $-1,854$ & 0,064 & & & \\
\hline Jakob1 2008 & Frozen ET & 0,091 & 0,023 & 0,300 & $-3,105$ & 0,002 & & & \\
\hline Jakob2 2011 & Frozen ET & 0,127 & 0,075 & 0,207 & $-6,479$ & 0,000 & & & \\
\hline Jakob3 2011 & Frozen ET & 0,182 & 0,114 & 0,276 & $-5,442$ & 0,000 & & & \\
\hline Jakob4 2011 & Frozen ET & 0,143 & 0,083 & 0,235 & $-5,746$ & 0,000 & & & \\
\hline Miyairi 2002 & Frozen ET & 0,105 & 0,026 & 0,337 & $-2,863$ & 0,004 & & & \\
\hline Mizuno 2003 & Frozen ET & 0,125 & 0,017 & 0,537 & $-1,820$ & 0,069 & & & \\
\hline Sakurai 2006 & Frozen ET & 0,021 & 0,001 & 0,259 & $-2,694$ & 0,007 & & & \\
\hline Shimamura1 2008 & Frozen ET & 0,056 & 0,027 & 0,112 & $-7,285$ & 0,000 & & & \\
\hline Shimamura2 2008 & Frozen ET & 0,072 & 0,030 & 0,163 & $-5,490$ & 0,000 & & & 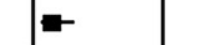 \\
\hline Sun 2011 & Frozen ET & 0,031 & 0,016 & 0,058 & $-10,173$ & 0,000 & & & \\
\hline Uchida.12006 & Frozen ET & 0,057 & 0,014 & 0,202 & $-3,850$ & 0,000 & & & $=-$ \\
\hline \multirow[t]{3}{*}{ Uchida.2 2010} & Frozen ET & 0,017 & 0,002 & 0,112 & $-4,008$ & 0,000 & & & $\vdash$ \\
\hline & & 0,098 & 0,080 & 0,119 & $-19,798$ & 0,000 & & & 1 \\
\hline & & & & & & & $-1,00$ & $-0,50$ & 0,00 \\
\hline
\end{tabular}

B

Perioperative mortality in stented elephant trunk

\begin{tabular}{|c|c|c|c|c|c|c|c|c|c|c|}
\hline \multirow[t]{2}{*}{ Study name } & \multirow[t]{2}{*}{ Mortality periop } & \multicolumn{4}{|c|}{ Statistics for each study } & & & \multicolumn{2}{|c|}{ Event rate and $95 \% \mathrm{Cl}$} & \\
\hline & & $\begin{array}{l}\text { Event } \\
\text { rate }\end{array}$ & $\begin{array}{l}\text { Lower } \\
\text { limit }\end{array}$ & $\begin{array}{l}\text { Upper } \\
\text { limit }\end{array}$ & Z-Value & p-Value & & & & \\
\hline Carroccio 2005 & Elephant trunk & 0,083 & 0,012 & 0,413 & $-2,296$ & 0,022 & & & & \\
\hline Greenberg 2005 & Elephant trunk & 0,045 & 0,006 & 0,261 & $-2,975$ & 0,003 & & & & \\
\hline Hughes.2 2009 & Elephant trunk & 0,063 & 0,004 & 0,539 & $-1,854$ & 0,064 & & & & \\
\hline $\operatorname{Jim} 2011$ & Elephant trunk & 0,100 & 0,014 & 0,467 & $-2,084$ & 0,037 & & & & \\
\hline Kawaharada 2009 & Elephant trunk & 0,016 & 0,001 & 0,206 & $-2,907$ & 0,004 & & & & \\
\hline Kieffer 2005 & Elephant trunk & 0,250 & 0,097 & 0,508 & $-1,903$ & 0,057 & & & & \\
\hline Lee.2 2011 & Elephant trunk & 0,190 & 0,073 & 0,412 & $-2,604$ & 0,009 & & & & \\
\hline Obitsu.2 2011 & Elephant trunk & 0,028 & 0,002 & 0,322 & $-2,479$ & 0,013 & & & & \\
\hline \multirow[t]{3}{*}{ Tse 2004} & Elephant trunk & 0,200 & 0,027 & 0,691 & $-1,240$ & 0,215 & & & & \\
\hline & & 0,132 & 0,078 & 0,214 & $-6,321$ & 0,000 & & & & \\
\hline & & & & & & & $-1,00$ & $-0,50$ & 0,00 & 1,00 \\
\hline
\end{tabular}

FIGURE 2. (continued).

4.3\% (95\% CI, 3.0-6.3) and spinal cord ischemia ranged from $0.0 \%$ to $12.2 \%$ with a pooled event ratio of $5.8 \%$ (95\% CI, 4.2-7.9).
Analyses by publication year. When breakdown of study by publication year (2007) was applied, pooled results showed no decreased mortality rates and a tendency for 


\section{Perioperative stroke in debranching}

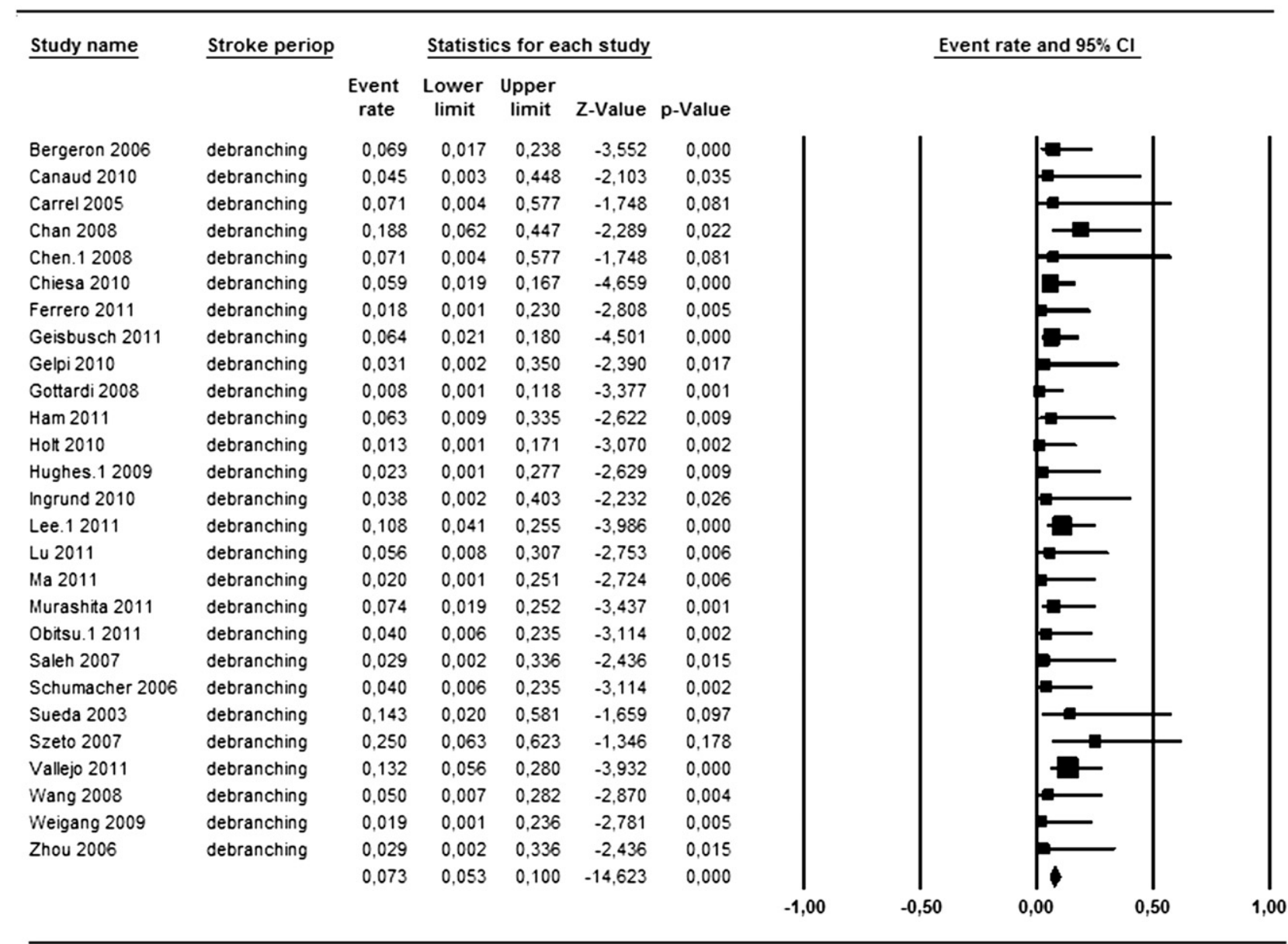

A

FIGURE 3. Perioperative stroke in hybrid arch repair. A, Debranching technique. B, Frozen elephant trunk technique. C, Stented elephant trunk technique. $C I$, Confidence interval; ET, elephant trunk.

lower neurologic complication in most recent series, but the numbers in each subgroup were smaller. In the 16 studies published before 2007, pooled mortality ratio was $11.4 \%$ (95\% CI, 7.9-16.2), stroke $9.9 \%$ (95\% CI, 6.4-15.1), and spinal cord ischemia $12.7 \%$ (95\% CI, 8.7-18.4); in studies published since 2007, pooled ratios were $10.7 \%(95 \% \mathrm{CI}, 8.1-12.5)$ for mortality, $6.4 \%$ (95\% CI 5.2-7.9) for stroke, and 5.6\% (95\% CI 4.57.0) for spinal cord ischemia. Specifically for dissection cases, pooled mortality, stroke, and spinal ischemia rates were $7.2 \%(95 \% \mathrm{CI}, 2.7-17.7), 5.8 \%(95 \% \mathrm{CI}$, $1.1-24.9)$, and $2.8 \%(95 \% \mathrm{CI}, 4.0-17.2)$ in old studies and $10.0 \% \quad(95 \% \quad \mathrm{CI}, \quad 7.8-12.8), \quad 4.2 \% \quad(95 \% \quad \mathrm{CI}$, 2.9-6.2), and 5.9\% (95\% CI, 4.3-8.1), respectively, in recent studies.

For debranching subgroups, pooled mortality, stroke, and spinal cord ischemia were $12.7 \%, 6.5 \%$, and $5.1 \%$ in old studies and $11.8 \%, 7.4 \%$, and $4.2 \%$ in recent studies; for the frozen elephant trunk group, pooled mortality, stroke, and spinal cord ischemia were $8.5 \%, 8.8 \%$, and $16.4 \%$ in older studies and $10 \%, 5.9 \%$, and $6.3 \%$ in recent studies; for the stented subgroup, pooled mortality, stroke, and spinal cord ischemia were $15.8 \%, 18.8 \%$, and $10.5 \%$ in older studies and $11.0 \%, 5.6 \%$, and $4.4 \%$ in recent studies.

Analyses by volume. To assess whether higher volumes affected the results, we analyzed studies with low volume $(<20)$ and those with larger volume $(\geq 20)$ separately. Higher volume seemed to decrease the neurologic complication, especially stroke, but no mortality rates. Pooled ratios of mortality, stroke, and spinal cord ischemia were $11.4 \%$ (95\% CI, 7.9-16.3), 11.2\% (95\% CI, 7.4-16.6), and $8.9 \%(95 \%$ CI, 5.6-13.8) in the 22 studies at low volume and $10.7 \%(95 \% \mathrm{CI}, 9.1-12.5), 6.1 \%(95 \% \mathrm{CI}$, 5.0-7.6), and 6.4\% (95\% CI, 5.2-7.9), respectively, in 
Perioperative stroke in frozen elephant trunk

\begin{tabular}{|c|c|c|c|c|c|c|c|c|}
\hline \multirow[t]{2}{*}{ Study name } & \multirow[t]{2}{*}{ Stroke periop } & \multicolumn{5}{|c|}{ Statistics for each study } & & Event rate and $95 \% \mathrm{Cl}$ \\
\hline & & $\begin{array}{c}\text { Event } \\
\text { rate }\end{array}$ & $\begin{array}{l}\text { Lower } \\
\text { limit }\end{array}$ & $\begin{array}{l}\text { Upper } \\
\text { limit }\end{array}$ & Z-Value & p-Value & & \\
\hline Baraki 2007 & Frozen ET & 0,128 & 0,054 & 0,273 & $-4,002$ & 0,000 & & \\
\hline Chavan 2005 & Frozen ET & 0,045 & 0,006 & 0,261 & $-2,975$ & 0,003 & & \\
\hline Chen.2 2010 & Frozen ET & 0,107 & 0,035 & 0,284 & $-3,470$ & 0,001 & & \\
\hline Di Eusanio 2011 & Frozen ET & 0,010 & 0,001 & 0,141 & $-3,233$ & 0,001 & & \\
\hline Fleck 2002 & Frozen ET & 0,125 & 0,017 & 0,537 & $-1,820$ & 0,069 & & \\
\hline Flores 2006 & Frozen ET & 0,160 & 0,061 & 0,357 & $-3,040$ & 0,002 & & \\
\hline Gorlitzer 2007 & Frozen ET & 0,143 & 0,020 & 0,581 & $-1,659$ & 0,097 & & \\
\hline Jakob1 2008 & Frozen ET & 0,091 & 0,023 & 0,300 & $-3,105$ & 0,002 & & \\
\hline Jakob2 2011 & Frozen ET & 0,029 & 0,010 & 0,087 & $-5,966$ & 0,000 & & \\
\hline Jakob3 2011 & Frozen ET & 0,057 & 0,024 & 0,129 & $-6,101$ & 0,000 & & \\
\hline Jakob4 2011 & Frozen ET & 0,095 & 0,048 & 0,179 & $-6,057$ & 0,000 & & \\
\hline Miyairi 2002 & Frozen ET & 0,025 & 0,002 & 0,298 & $-2,558$ & 0,011 & & \\
\hline Mizuno 2003 & Frozen ET & 0,125 & 0,017 & 0,537 & $-1,820$ & 0,069 & & \\
\hline Sakurai 2006 & Frozen ET & 0,043 & 0,006 & 0,252 & $-3,023$ & 0,003 & & \\
\hline Shimamura1 2008 & Frozen ET & 0,056 & 0,027 & 0,112 & $-7,285$ & 0,000 & & \\
\hline Shimamura2 2008 & Frozen ET & 0,058 & 0,022 & 0,144 & $-5,412$ & 0,000 & & \\
\hline Sun 2011 & Frozen ET & 0,024 & 0,012 & 0,050 & $-9,679$ & 0,000 & & \\
\hline Uchida.12010 & Frozen ET & 0,034 & 0,009 & 0,128 & $-4,630$ & 0,000 & & - \\
\hline \multirow[t]{3}{*}{ Uchida.2 2006} & Frozen ET & 0,014 & 0,001 & 0,187 & $-2,993$ & 0,003 & & \\
\hline & & 0,062 & 0,048 & 0,080 & $-19,575$ & 0,000 & & 1 \\
\hline & & & & & & & $-1,00$ & $-0,50$ \\
\hline
\end{tabular}

B

Perioperative stroke in stented elephant trunk

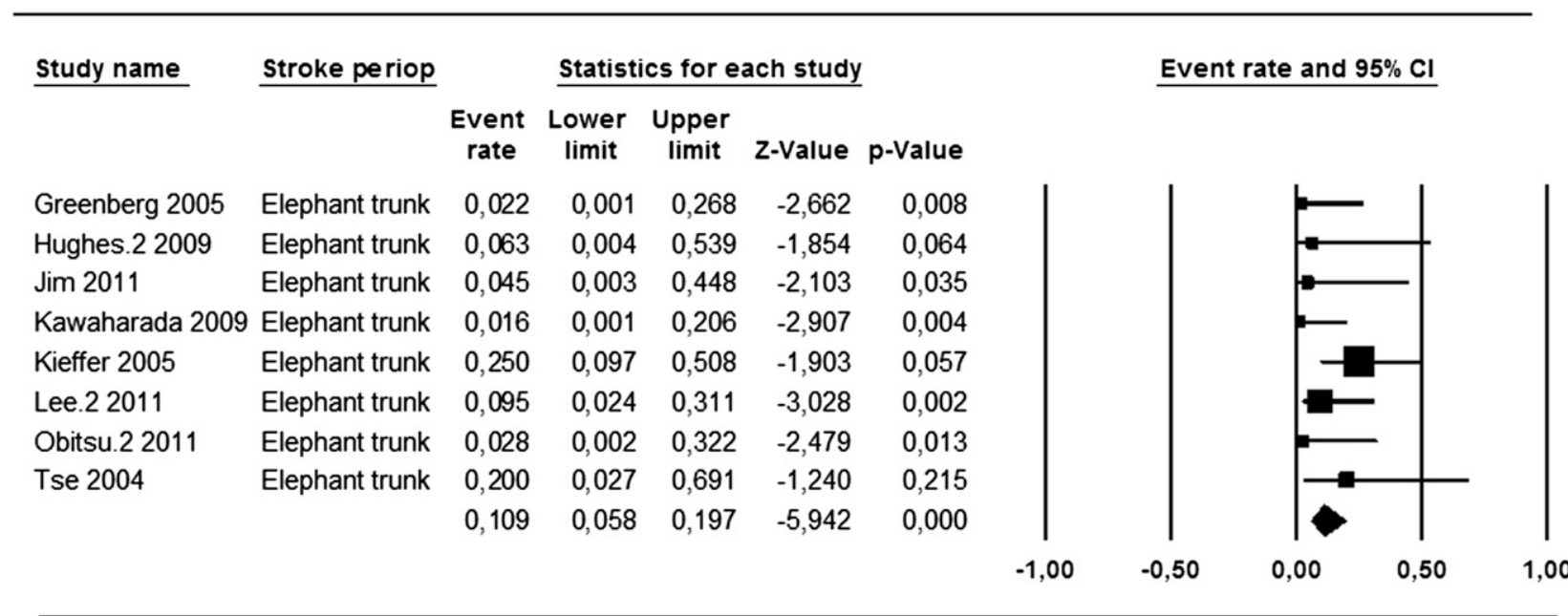

C

FIGURE 3. (continued).

the remaining studies at higher volume. Specifically, in the dissection subgroup, pooled mortality stroke and spinal cord ischemia were 9.1\% (95\% CI, 4.4-17.9), 7.0\% (95\% CI, 2.7-17.3), and 4.6\% (95\% CI, 1.5-13.4) in low volume studies and 9.9\% (95\% CI, 7.7-12.7), 4.0\%
(95\% CI, 2.6-5.9), and 5.9\% (95\% CI, 4.3-8.1) in higher volume studies.

For the debranching subgroup, pooled mortality, stroke, and spinal cord ischemia were $10.2 \%, 9.6 \%$, and $5.6 \%$ in low volume studies and $12.5 \%, 6.5 \%$, and $3.8 \%$ in 


\section{Perioperative spinal cord ischemia in debranching}

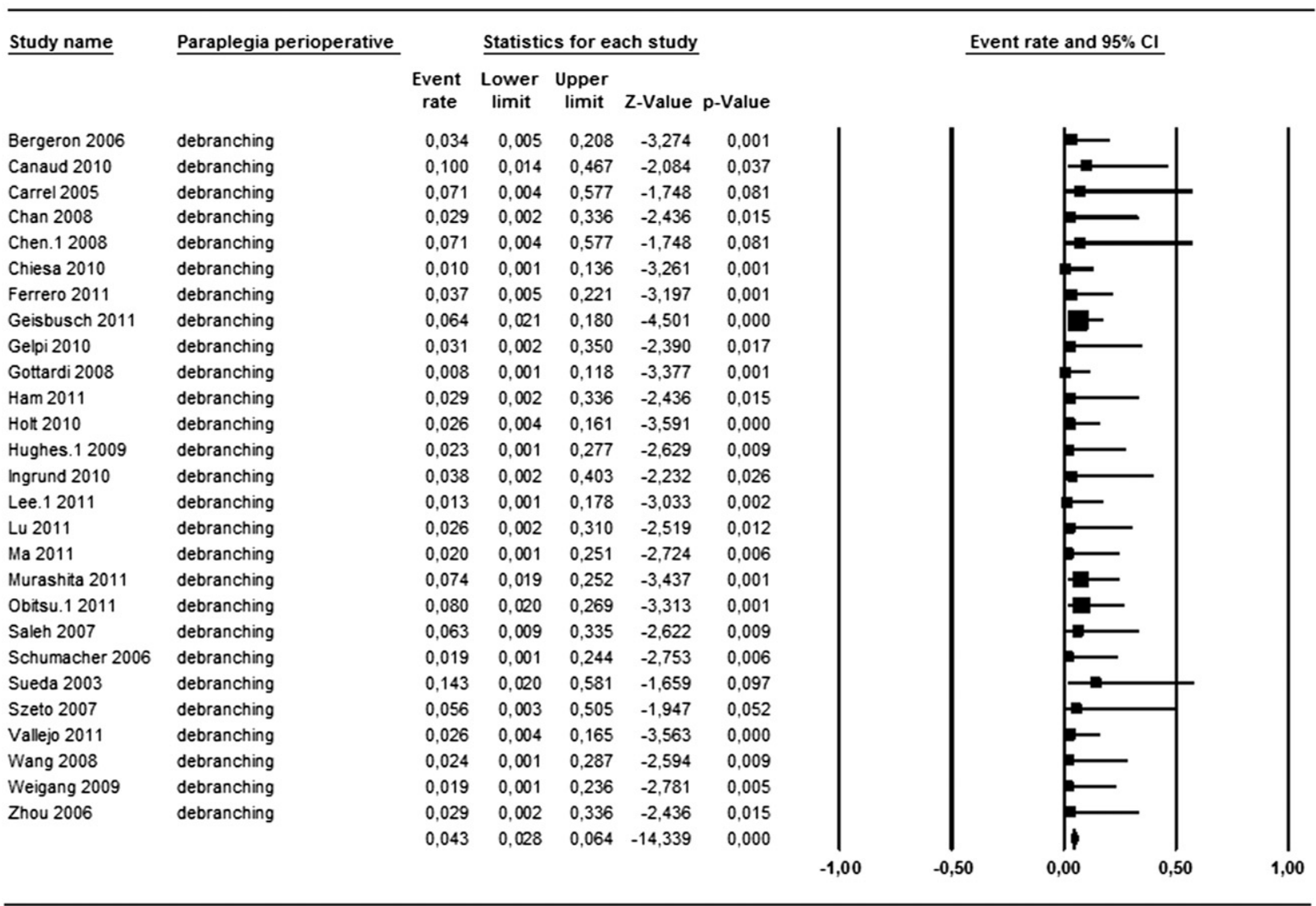

A

FIGURE 4. Perioperative spinal cord ischemia in hybrid arch repair. A, Debranching technique. B, Frozen elephant trunk technique. C, Stented elephant trunk technique. $C I$, Confidence interval; ET, elephant trunk.

higher volume studies; for the frozen elephant trunk group, pooled mortality, stroke, and spinal cord ischemia were $10.7 \%, 10.2 \%$, and $18.2 \%$ in low volume studies and $9.8 \%, 6.1 \%$, and $7.3 \%$ in higher volume centers; for the stented subgroup, pooled mortality, stroke, and spinal cord ischemia were $14.7 \%, 15.5 \%$, and $6.2 \%$ in low volume studies and $11.3 \%, 5.4 \%$, and $8.1 \%$ in higher volume centers.

When both the cutoff values (publication year and volume) were applied, similar results were achieved inasmuch as most old studies were at low volume.

Details for subgroup analyses by year and volume are provided in Appendixes E1 and E2.

Zones (Figure 6, $\boldsymbol{A}-\boldsymbol{C}$ ). A secondary analysis was performed according to landing zone 0 versus 1 for hybrid arch procedures. For the present analysis, zones 2 or more were excluded. Data were available from 19 studies including 187 patients with landing zones 0 and 202 with landing zone 1. 2,13,14,E21-E28,E30,E31,E33,E34,E36,E37,E41,E53
Pooled perioperative mortality was $15.1 \%(95 \% \mathrm{CI}$, 10.3-21.5) for landing zone 0 and 7.6\% (95\% CI, 4.33.0) for landing zone 1 , resulting in a significant higher risk for zone $0(\mathrm{OR}, 2.8 ; 95 \% \mathrm{CI}, 1.17-6.7 ; P=.021$; Figure 6, A).

Pooled perioperative stroke was $7.1 \%(95 \% \mathrm{CI}$, 4.1-12.2) for zone 0 and $10.8 \%$ (95\% CI, 6.4-17.7) for zone 1 , resulting in a nonsignificant difference between zones (OR, $0.45 ; 95 \% \mathrm{CI}, 0.13-1.54 ; P=.20$; Figure 6, $B$ ).

Pooled spinal cord ischemia was $6.5 \%(95 \% \mathrm{CI}, 3.5-$ 11.8 ) in zone 0 and $5.2 \%$ (95\% CI, 2.7-9.8) in zone 1 , resulting in a nonsignificant difference between zones (OR, 1.43; 95\% CI, 0.32-6.47; $P=.63$; (Figure 6, $C$ ).

\section{DISCUSSION}

Although in the recent years hybrid repair appears to be an appealing procedure for the treatment of aortic arch diseases in selected patients, the technique has not been validated as a treatment option and this literature search did 
Perioperative spinal cord ischemia in frozen elephant trunk

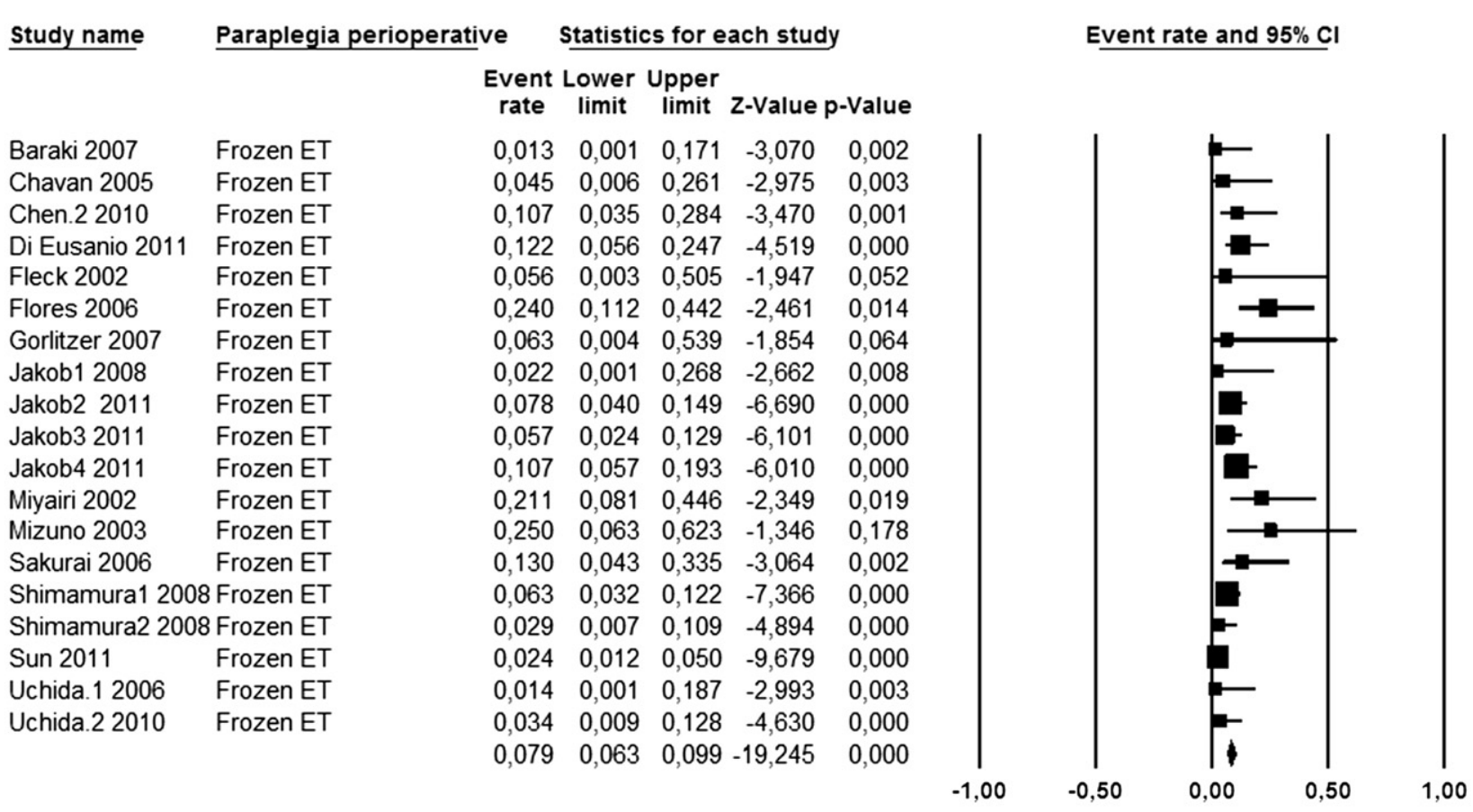

B

Perioperative spinal cord ischemia in stented elephant trunk

\begin{tabular}{|c|c|c|c|c|c|c|c|c|c|c|}
\hline \multirow[t]{2}{*}{ Study name } & \multicolumn{2}{|l|}{ Paraplegia perioperative } & \multicolumn{4}{|c|}{ Statistics for each study } & \multicolumn{4}{|c|}{ Event rate and $95 \% \mathrm{Cl}$} \\
\hline & & $\begin{array}{l}\text { Event } \\
\text { rate }\end{array}$ & $\begin{array}{l}\text { Lower } \\
\text { limit }\end{array}$ & $\begin{array}{l}\text { Upper } \\
\text { limit }\end{array}$ & Z-Value $p$ & -Value & & & & \\
\hline Greenberg 2005 & Elephant trunk & 0,136 & 0,045 & 0,348 & $-2,971$ & 0,003 & & & & \\
\hline Hughes.2 2009 & Elephant trunk & 0,143 & 0,020 & 0,581 & $-1,659$ & 0,097 & & & & \\
\hline $\operatorname{Jim} 2011$ & Elephant trunk & 0,045 & 0,003 & 0,448 & $-2,103$ & 0,035 & & & & \\
\hline Kawaharada 2009 & Elephant trunk & 0,016 & 0,001 & 0,206 & $-2,907$ & 0,004 & & & & \\
\hline Kieffer 2005 & Elephant trunk & 0,029 & 0,002 & 0,336 & $-2,436$ & 0,015 & & & & \\
\hline Lee.2 2011 & Elephant trunk & 0,023 & 0,001 & 0,277 & $-2,629$ & 0,009 & & & & \\
\hline Obitsu.2 2011 & Elephant trunk & 0,028 & 0,002 & 0,322 & $-2,479$ & 0,013 & & & & \\
\hline \multirow[t]{3}{*}{ Tse 2004} & Elephant trunk & 0,083 & 0,005 & 0,622 & $-1,623$ & 0,105 & & & & \\
\hline & & 0,072 & 0,035 & 0,145 & $-6,425$ & 0,000 & & & & \\
\hline & & & & & & & $-1,00$ & $-0,50$ & 0,50 & 1,00 \\
\hline
\end{tabular}

FIGURE 4. (continued).

not identify prospective randomized or large nonrandomized studies comparing hybrid arch procedures with other conventional or innovative treatment strategies for aortic arch repair.

This review specifically focused on outcomes of patients receiving hybrid arch repair for aortic dissection. As reported by pooled analysis of 17 studies $^{2,5-20}$ hybrid arch procedures in dissected aorta can be performed with mortality risks (ranging from $0.0 \%$ to $14.3 \%$ with a pooled event ratio of $9.8 \%$ ) similar to other hybrid arch repairs but with relatively low periprocedural neurologic risks (pooled stroke incidence, 4.3\%; pooled spinal cord ischemia incidence, 5.8\%). High volume centers and more recent experiences may have influenced decreased stroke rates. These data may be even more relevant because they included high-risk patients and 


\section{Perioperative mortality in dissection}

\begin{tabular}{|c|c|c|c|c|c|c|c|c|c|}
\hline \multirow[t]{2}{*}{ Study name } & \multicolumn{5}{|c|}{ Statistics for each study } & & \multicolumn{2}{|c|}{ Event rate and $95 \% \mathrm{Cl}$} & \\
\hline & $\begin{array}{l}\text { Event } \\
\text { rate }\end{array}$ & $\begin{array}{c}\text { Lower } \\
\text { limit }\end{array}$ & $\begin{array}{l}\text { Upper } \\
\text { limit }\end{array}$ & Z-Value & p-Value & & & & \\
\hline Baraki 2007 & 0,143 & 0,047 & 0,361 & $-2,873$ & 0,004 & & & & \\
\hline Chavan 2005 & 0,067 & 0,009 & 0,352 & $-2,550$ & 0,011 & & & & \\
\hline Chen 2010 & 0,143 & 0,055 & 0,324 & $-3,318$ & 0,001 & & & & \\
\hline Di Eusanio 2011 & 0,102 & 0,043 & 0,223 & $-4,608$ & 0,000 & & & & \\
\hline Fleck 2002 & 0,125 & 0,017 & 0,537 & $-1,820$ & 0,069 & & & & \\
\hline Gorlitzer 2007 & 0,083 & 0,005 & 0,622 & $-1,623$ & 0,105 & & & & \\
\hline Ingrund 2010 & 0,111 & 0,015 & 0,500 & $-1,961$ & 0,050 & & & & \\
\hline Jakob1 2008 & 0,091 & 0,023 & 0,300 & $-3,105$ & 0,002 & & & & \\
\hline Jakob2 2011 & 0,127 & 0,075 & 0,207 & $-6,479$ & 0,000 & & & & \\
\hline Jakob3 2011 & 0,182 & 0,114 & 0,276 & $-5,442$ & 0,000 & & & & \\
\hline Lu 2011 & 0,111 & 0,028 & 0,352 & $-2,773$ & 0,006 & & & & \\
\hline Ma 2011 & 0,020 & 0,001 & 0,251 & $-2,724$ & 0,006 & & & & \\
\hline Obitsu 2011 & 0,050 & 0,003 & 0,475 & $-2,029$ & 0,042 & & & & \\
\hline Shimamura1 2008 & 0,035 & 0,009 & 0,130 & $-4,604$ & 0,000 & & & & \\
\hline Sun 2011 & 0,031 & 0,016 & 0,058 & $-10,173$ & 0,000 & & & & \\
\hline Uchida 2006 & 0,057 & 0,014 & 0,202 & $-3,850$ & 0,000 & & & $=-$ & \\
\hline \multirow[t]{3}{*}{ Wang 2008} & 0,071 & 0,010 & 0,370 & $-2,472$ & 0,013 & & & & \\
\hline & 0,098 & 0,077 & 0,124 & $-16,613$ & 0,000 & & & 1 & \\
\hline & & & & & & $-1,00$ & $-0,50$ & 0,00 & 1,00 \\
\hline
\end{tabular}

A

FIGURE 5. Perioperative results in hybrid arch repair for aortic dissection. A, Perioperative mortality. B, Perioperative stroke. C, Perioperative spinal cord ischemia. $C I$, Confidence interval.

emergency cases. Unfortunately, there were no detailed outcomes for type A/B dissection or chronic/acute cases, neither risk nor timing stratification to provide more detailed information.

Our analysis showed that the overall hybrid arch procedures were associated with about $10 \%$ periprocedural mortality and about 7\% periprocedural risk of stroke or spinal cord ischemia. Mortality was not affected by increased volume center and time of experience, whereas neurologic complications were positively affected: in studies with fewer than 20 cases, pooled stroke rates of $11.2 \%$ and spinal cord ischemia rates of $8.9 \%$ were found, whereas in studies with larger case loads, the pooled rates were $6.1 \%$ for stroke and $6.4 \%$ for spinal cord ischemia. Similarly, pooled stroke and spinal cord ischemia rates were $9.9 \%$ and $12.7 \%$ in older studies while $6.4 \%$ and $5.6 \%$, respectively, in studies published in 2007 or after. Furthermore, we measured all the spinal cord ischemia occurring in the periprocedural period (30 days or in-hospital) and included permanent and temporary events, thereby leading to potential overestimation of risks.
However, different techniques for different disease extension and patient settings have been used in hybrid arch repair, and the clinical outcomes have not always been separately reported, making it difficult to compare or pool the results across studies. To make results more comparable, we separately analyzed hybrid arch procedures (regardless of the aortic disease) consisting in aortic arch "debranching," "frozen elephant trunk," and "stented elephant trunk" using the classification suggested by Eagleton and Greenberg ${ }^{1}$ and Szeto and Bavaria. ${ }^{\text {E78 }}$ However, large heterogeneity still remained.

Our data on outcomes of hybrid arch repairs are in similar ranges of series published during the past 10 years for open arch repairs (references online only). ${ }^{\text {E54-E60 }}$ Although better results have been published in selected experiences, ${ }^{\mathrm{E} 59, \mathrm{E} 60}$ open surgery for the aortic arch remains a very challenging procedure in many common centers.

From this review, the pooled stroke incidence during hybrid arch repair was not negligible but affected by volume and experience: in the debranching group the rates were $9.6 \%$ in low volume and $6.5 \%$ in high 
Perioperative stroke in dissection

\begin{tabular}{|c|c|c|c|c|c|c|c|c|c|c|}
\hline \multirow[t]{2}{*}{ Study name } & \multicolumn{5}{|c|}{ Statistics for each study } & \multicolumn{5}{|c|}{ Event rate and $95 \% \mathrm{Cl}$} \\
\hline & $\begin{array}{l}\text { Event } \\
\text { rate }\end{array}$ & $\begin{array}{c}\text { Lower } \\
\text { limit }\end{array}$ & $\begin{array}{l}\text { Upper } \\
\text { limit }\end{array}$ & Z-Value & p-Value & & & & & \\
\hline Chen 2010 & 0,107 & 0,035 & 0,284 & $-3,470$ & 0,001 & & & & & \\
\hline Di Eusanio 2011 & 0,010 & 0,001 & 0,141 & $-3,233$ & 0,001 & & & & & \\
\hline Fleck 2002 & 0,125 & 0,017 & 0,537 & $-1,820$ & 0,069 & & & & & \\
\hline Ingrund 2010 & 0,050 & 0,003 & 0,475 & $-2,029$ & 0,042 & & & & & \\
\hline Jakob1 2008 & 0,091 & 0,023 & 0,300 & $-3,105$ & 0,002 & & & & & \\
\hline Jakob2 2011 & 0,029 & 0,010 & 0,087 & $-5,966$ & 0,000 & & & & & \\
\hline Jakob3 2011 & 0,057 & 0,024 & 0,129 & $-6,101$ & 0,000 & & & & & \\
\hline Lu 2011 & 0,056 & 0,008 & 0,307 & $-2,753$ & 0,006 & & & & & \\
\hline Ma 2011 & 0,020 & 0,001 & 0,251 & $-2,724$ & 0,006 & & & & & \\
\hline Obitsu 2011 & 0,050 & 0,003 & 0,475 & $-2,029$ & 0,042 & & & & & \\
\hline Shimamura 22008 & 0,030 & 0,004 & 0,186 & $-3,413$ & 0,001 & & & & & \\
\hline Sun 2011 & 0,024 & 0,012 & 0,050 & $-9,679$ & 0,000 & & & & & \\
\hline Uchida 2006 & 0,014 & 0,001 & 0,187 & $-2,993$ & 0,003 & & & & & \\
\hline \multirow[t]{3}{*}{ Wang 2008} & 0,071 & 0,010 & 0,370 & $-2,472$ & 0,013 & & & & & \\
\hline & 0,043 & 0,030 & 0,063 & $-15,527$ & 0,000 & & & | & & \\
\hline & & & & & & $-1,00$ & $-0,50$ & 0,00 & 0,50 & 1,00 \\
\hline
\end{tabular}

B

Perioperative spinal cord ischemia in dissection

\begin{tabular}{|c|c|c|c|c|c|c|c|c|c|c|}
\hline \multirow[t]{2}{*}{ Study name } & \multicolumn{5}{|c|}{ Statistics for each study } & & \multicolumn{4}{|c|}{ Event rate and $95 \% \mathrm{Cl}$} \\
\hline & $\begin{array}{l}\text { Event } \\
\text { rate }\end{array}$ & $\begin{array}{c}\text { Lower } \\
\text { limit }\end{array}$ & $\begin{array}{l}\text { Upper } \\
\text { limit }\end{array}$ & Z-Value $p$ & -Value & & & & & \\
\hline Baraki 2007 & 0,023 & 0,001 & 0,277 & $-2,629$ & 0,009 & & & & & \\
\hline Chen 2010 & 0,107 & 0,035 & 0,284 & $-3,470$ & 0,001 & & & & & \\
\hline Di Eusanio 2011 & 0,122 & 0,056 & 0,247 & $-4,519$ & 0,000 & & & & & \\
\hline Fleck 2002 & 0,056 & 0,003 & 0,505 & $-1,947$ & 0,052 & & & & & \\
\hline Gorlitzer 2007 & 0,083 & 0,005 & 0,622 & $-1,623$ & 0,105 & & & & & \\
\hline Ingrund 2010 & 0,050 & 0,003 & 0,475 & $-2,029$ & 0,042 & & & & & \\
\hline Jakob1 2008 & 0,022 & 0,001 & 0,268 & $-2,662$ & 0,008 & & & & & \\
\hline Jakob2 2011 & 0,078 & 0,040 & 0,149 & $-6,690$ & 0,000 & & & & & \\
\hline Jakob3 2011 & 0,057 & 0,024 & 0,129 & $-6,101$ & 0,000 & & & & & \\
\hline Lu 2011 & 0,026 & 0,002 & 0,310 & $-2,519$ & 0,012 & & & & & \\
\hline Ma 2011 & 0,020 & 0,001 & 0,251 & $-2,724$ & 0,006 & & & & & \\
\hline Obitsu 2011 & 0,050 & 0,003 & 0,475 & $-2,029$ & 0,042 & & & & & \\
\hline Shimamura1 2008 & 0,070 & 0,027 & 0,173 & $-4,983$ & 0,000 & & & & & \\
\hline Sun 2011 & 0,024 & 0,012 & 0,050 & $-9,679$ & 0,000 & & & & & \\
\hline Uchida 2006 & 0,014 & 0,001 & 0,187 & $-2,993$ & 0,003 & & & & & \\
\hline \multirow[t]{3}{*}{ Wang 2008} & 0,033 & 0,002 & 0,366 & $-2,341$ & 0,019 & & & & & \\
\hline & 0,058 & 0,042 & 0,079 & $-16,590$ & 0,000 & & & 1 & & \\
\hline & & & & & & $-1,00$ & $-0,50$ & 0,00 & 0,50 & 1,00 \\
\hline
\end{tabular}

C

FIGURE 5. (continued). 


\section{Perioperative mortality by zone}

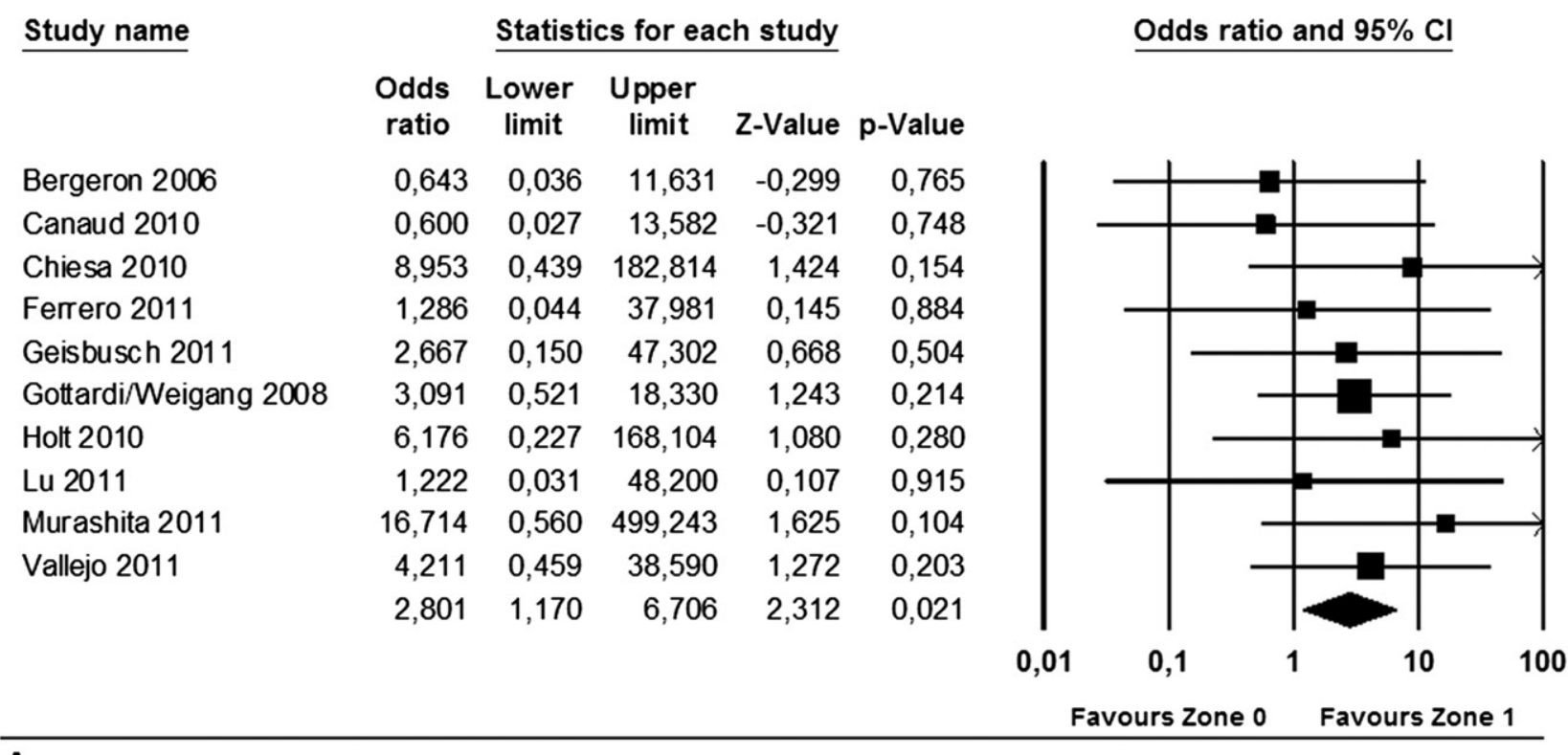

A

FIGURE 6. Perioperative results in hybrid repairs by landing zone (zone 0 vs zone 1) A, Perioperative mortality. B, Perioperative stroke. C, Perioperative spinal cord ischemia. $C I$, Confidence interval.

volume centers. In the frozen group, pooled stroke rates were $10.2 \%$ and $8.8 \%$ in low volume and older studies, whereas rates were $6.1 \%$ and $5.9 \%$ in higher volume and recent studies. Similarly, for stented elephant trunk, stroke rates of $15.5 \%$ and $18.8 \%$ were detected in low volume and old studies, and rates of $5.4 \%$ and $5.6 \%$ in higher volume and recent studies

Similarly to other reviews that analyzed total versus partial debranching stroke risk, ${ }^{\text {E76,E79 }}$ we stratified the results according to landing zones: our review confirmed 2.8 higher mortality risks for total debranching (landing zone 0) but did not show significant differences in the neurologic risks of stroke or spinal cord ischemia. Analyses by zone could not be stratified by year and volume because of the small numbers.

This review has several limitations. Although it is one of the largest reviews published to date regarding hybrid arch repair, the pooled results are weakened because of large heterogeneity in techniques and lack of standardization in reporting patients' specific data and end points. Even though data were separately extracted and analyzed by 3 different operative techniques, we were unable to perform detailed analyses on patients and technique risk stratification. Furthermore, we specifically focused the review on periprocedural clinical outcome and did not assess technical feasibility and long-term results. However, feasibility data were indeed more inconsistently published across studies and pooled analysis would have provided limited information. Finally, some small sample size studies were included whereas a larger number of patients is needed to better identify statistically significant differences. However, breakdown of data by volume center was performed to exclude the smaller series from calculations.

In conclusion, hybrid repair of aortic arch disease presents a persistent high risk of perioperative mortality, comparable with open repair. However, rates of neurologic complications seem to have decreased with timing and center volume and may be low in dissection cases: $4.3 \%$ periprocedural stroke, $5.8 \%$ spinal cord ischemia. Hybrid arch repair involving the ascending aorta (zone 0) presents almost 3 times higher mortality than repair involving zone 1 , although the incidence of neurologic complications may be similar.

Nevertheless, no reliable long-term data exist to ascertain the durability of the hybrid arch procedure, and contemporary conclusions are mainly provided from relatively small case series or retrospective studies. Further research is required to strengthen outcomes of this treatment, and ideally technologic improvements may be made in the next years to help to further improve the outcomes of aortic arch repair. 


\section{Perioperative stroke by zone}

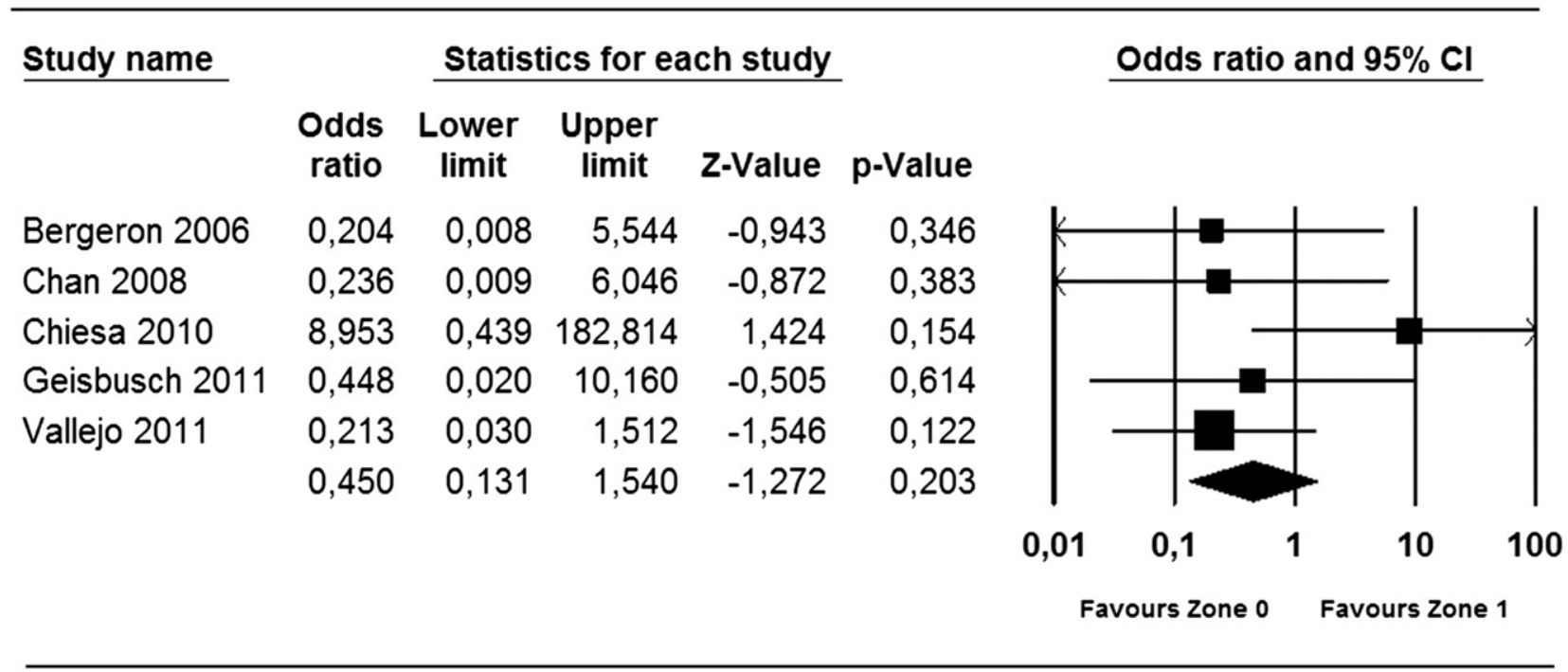

B

\section{Perioperative spinal cord ischemia by zone}

\section{Study name}

$\begin{array}{lcccrrr} & \begin{array}{r}\text { Odds } \\ \text { ratio }\end{array} & \begin{array}{c}\text { Lower } \\ \text { limit }\end{array} & \begin{array}{c}\text { Upper } \\ \text { limit }\end{array} & \text { Z-Value } & \text { p-Value } \\ \text { Canaud 2010 } & 2,455 & 0,079 & 76,132 & 0,512 & 0,608 \\ \text { Geisbusch 2011 } & 0,778 & 0,029 & 20,696 & -0,150 & 0,881 \\ \text { Holt 2010 } & 0,579 & 0,021 & 15,676 & -0,325 & 0,745 \\ \text { Hughes 2 2009 } & 5,400 & 0,154 & 188,831 & 0,930 & 0,352 \\ \text { Vallejo 2011 } & 1,302 & 0,049 & 34,412 & 0,158 & 0,875 \\ & 1,438 & 0,320 & 6,473 & 0,474 & 0,636\end{array}$

\section{Odds ratio and $95 \% \mathrm{Cl}$}

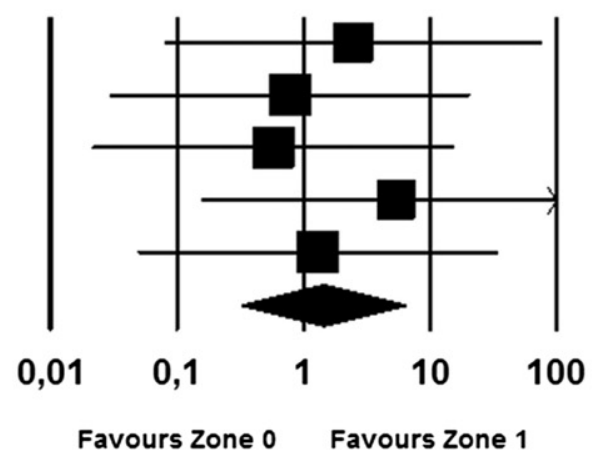

$\bar{C}$

FIGURE 6. (continued).

\section{References}

1. Eagleton MJ, Greenberg RK. Hybrid procedures for the treatment of aortic arch aneurysms. J Cardiovasc Surg (Torino). 2010;51:807-19.

2. Ingrund JC, Nasser F, Jesus-Silva SG, Limaco RP, Galastri FL, Burihan MC, et al. Hybrid procedures for complex thoracic aortic diseases. Rev Bras Cir Cardiovasc. 2010;25:303-10.

3. Mitchell RS, Ishimaru S, Ehrlich MP, Iwase T, Lauterjung L, Shimono T, et al. First international summit on thoracic aortic endografting: round table on thoracic aortic dissection as an indication for endografting. $J$ Endovasc Ther. 2002;9(Suppl 2):II98-105.

4. Harris RP, Helfand M, Woolf SH, Lohr KN, Mulrow CD, Teutsch SM, et al, Methods Work Group, Third US Preventive Services Task Force. Current methods of the US Preventive Services Task Force: a review of the process. Am J Prev Med. 2001;20(3 Suppl):21-35.
5. Baraki H, Hagl C, Khaladj N, Kallenbach K, Weidemann J, Haverich A, et al. The frozen elephant trunk technique for treatment of thoracic aortic aneurysms. Ann Thorac Surg. 2007;83:S819-23.

6. Chavan A, Karck M, Hagl C, Winterhalter M, Baus S, Galanski M, et al. Hybrid endograft for one-step treatment of multisegment disease of the thoracic aorta. $J$ Vasc Interv Radiol. 2005;16:823-9.

7. Chen X, Huang F, Xu M, Wang L, Jiang Y, Xiao L, et al. The stented elephant trunk procedure combined total arch replacement for Debakey I aortic dissection: operative result and follow-up. Interact Cardiovasc Thorac Surg. 2010; 11:594-8.

8. Di Eusanio M, Armaro A, Di Marco L, Pacini D, Savini C, Suarez SM, et al. Short- and midterm results after hybrid treatment of chronic aortic dissection with the frozen elephant trunk technique. Eur J Cardiothorac Surg. 2011;40: 875-80. 
9. Fleck T, Hutschala D, Czerny M, Ehrlich MP, Kasimir MT, Cejna M, et al. Combined surgical and endovascular treatment of acute aortic dissection type A: preliminary results. Ann Thorac Surg. 2002;74:761-5.

10. Gorlitzer M, Weiss G, Thalmann M, Mertikian G, Wislocki W, Meinhart J, et al. Combined surgical and endovascular repair of complex aortic pathologies with a new hybrid prosthesis. Ann Thorac Surg. 2007; 84:1971-6.

11. Jakob H, Tsagakis K, Tossios P, Massoudy P, Thielmann M, Buck T, et al. Combining classic surgery with descending stent grafting for acute DeBakey type I dissection. Ann Thorac Surg. 2008;86:95-101.

12. Jakob H, Tsagakis K, Pacini D, Di Bartolomeo R, Mestres C, Mohr F, et al. The International E-vita Open Registry: data sets of 274 patients. J Cardiovasc Surg (Torino). 2011;52:717-23.

13. Lu Q, Jing Z, Zhao Z, Bao J, Feng X, Feng R, Mei Z. Endovascular stent graft repair of aortic dissection type B extending to the aortic arch. Eur J Vasc Endovasc Surg. 2011;42:456-63.

14. Ma H, Yang HY, Zou JJ, Zhang XW. Management with the insufficient proximal landing zone for endovascular repair in aortic dissection. Chin Med J (Engl). 2011;124:3003-7.
15. Obitsu Y, Koizumi N, Takahashi S, Iida Y, Saiki N, Watanabe Y, et al. Hybrid procedures combining conventional and thoracic endovascular aortic repair for thoracic aortic aneurysms. Surg Today. 2011;41:922-7.

16. Shimamura K, Kuratani T, Matsumiya G, Kato M, Shirakawa Y, Takano H, et al. Long-term results of the open stent-grafting technique for extended aortic arch disease. J Thorac Cardiovasc Surg. 2008;135:1261-9.

17. Shimamura K, Kuratani T, Matsumiya G, Shirakawa Y, Takeuchi M, Takano H, et al. Hybrid endovascular aortic arch repair using branched endoprosthesis: the second-generation "branched" open stent-grafting technique. J Thorac Cardiovasc Surg. 2009;138:46-52.

18. Sun L, Qi R, Zhu J, Liu Y, Zheng J. Total arch replacement combined with stented elephant trunk implantation: a new "standard" therapy for type A dissection involving repair of the aortic arch? Circulation. 2011;123:971-8.

19. Uchida N, Ishihara H, Shibamura H, Kyo Y, Ozawa M. Midterm results of extensive primary repair of the thoracic aorta by means of total arch replacement with open stent graft placement for an acute type A aortic dissection. J Thorac Cardiovasc Surg. 2006;131:862-7.

20. Wang S, Chang G, Li X, Hu Z, Li S, Yang J, et al. Endovascular treatment of arch and proximal thoracic aortic lesions. J Vasc Surg. 2008;48:64-8. 


\section{E-References: Included Studies}

\section{Debranching}

E21. Bergeron P, Coulon P, De Chaumaray T, Ruiz M, Mariotti F, Gay J, et al. Great vessels transposition and aortic arch exclusion. J Cardiovasc Surg (Torino). 2005;46:141-7.

E22. Canaud L, Hireche K, Berthet JP, Branchereau P, Marty-Ané C, Alric P. Endovascular repair of aortic arch lesions in high-risk patients or after previous aortic surgery: midterm results. J Thorac Cardiovasc Surg. 2010;140:52-8.

E23. Carrel TP, Do DD, Triller J, Schmidli J. A less invasive approach to completely repair the aortic arch. Ann Thorac Surg. 2005;80:1475-8.

E24. Chan YC, Cheng SW, Ting AC, Ho P. Supra-aortic hybrid endovascular procedures for complex thoracic aortic disease: single center early to midterm results. J Vasc Surg. 2008;48:571-9.

E25. Chen IM, Wu FY, Shih CC. Banding technique for endovascular repair of arch aneurysm with unsuitable proximal landing zone. Circ J. 2008;72:1981-5.

E26. Chiesa R, Melissano G, Tshomba Y, Civilini E, Marone EM, Bertoglio L, et al. Ten years of endovascular aortic arch repair. J Endovasc Ther. 2010;17:1-11.

E27. Ferrero E, Ferri M, Viazzo A, Robaldo A, Zingarelli E, Sansone F, et al. Is total debranching a safe procedure for extensive aortic-arch disease? A single experience of 27 cases. Eur J Cardiothorac Surg. 2012;41:177-82.

E28. Geisbüsch P, Kotelis D, Müller-Eschner M, Hyhlik-Dürr A, Böckler D. Complications after aortic arch hybrid repair. $J$ Vasc Surg. 2011;53:935-41

E29. Gelpi G, Vanelli P, Mangini A, Danna P, Contino M, Antona C. Hybrid aortic arch repair procedure: reinforcement of the aorta for a safe and durable landing zone. Eur J Vasc Endovasc Surg. 2010;40:709-14.

E30. Gottardi R, Funovics M, Eggers N, Hirner A, Dorfmeister M, Holfeld J, et al. Supra-aortic transposition for combined vascular and endovascular repair of aortic arch pathology. Ann Thorac Surg. 2008;86:1524-9.

E31. Weigang E, Parker J, Czerny M, Peivandi AA, Dorweiler B, Beyersdorf F, et al. Endovascular aortic arch repair after aortic arch de-branching. Ann Thorac Surg. 2009;87:603-7.

E32. Ham SW, Chong T, Moos J, Rowe VL, Cohen RG, Cunningham MJ, et al. Arch and visceral/renal debranching combined with endovascular repair for thoracic and thoracoabdominal aortic aneurysms. J Vasc Surg. 2011;54:30-40.

E33. Holt PJ, Johnson C, Hinchliffe RJ, Morgan R, Jahingiri M, Loftus IM, et al. Outcomes of the endovascular management of aortic arch aneurysm: implications for management of the left subclavian artery. J Vasc Surg. 2010;51:1329-38.

E34. Hughes GC, Daneshmand MA, Balsara KR, Achneck HA, Sileshi B, Lee SM, et al. "Hybrid" repair of aneurysms of the transverse aortic arch: midterm results. Ann Thorac Surg. 2009;88:1882-7.

E35. Lee CW, Beaver TM, Klodell CT Jr, Hess PJ Jr, Martin TD, Feezor RJ, et al. Arch debranching versus elephant trunk procedures for hybrid repair of thoracic aortic pathologies. Ann Thorac Surg. 2011;91:465-71.

E36. Murashita T, Matsuda H, Domae K, Iba Y, Tanaka H, Sasaki H, et al. Less invasive surgical treatment for aortic arch aneurysms in high-risk patients: a comparative study of hybrid thoracic endovascular aortic repair and conventional total arch replacement. J Thorac Cardiovasc Surg. 2012;143:1007-13.

E37. Saleh HM. Hybrid repair of aortic arch aneurysm. Acta Chir Belg. 2007;107: 173-80.

E38. Schumacher H, Von Tengg-Kobligk H, Ostovic M, Henninger V, Ockert S, Böckler D, et al. Hybrid aortic procedures for endoluminal arch replacement in thoracic aneurysms and type B dissections. J Cardiovasc Surg (Torino). 2006;47:509-17

E39. Sueda T, Orihashi K, Okada K, Sugawara Y, Imai K, Kochi K. Fate of aneurysms of the distal arch and proximal descending thoracic aorta after transaortic endovascular stent-grafting. Ann Thorac Surg. 2003;76:84-9.

E40. Szeto WY, Bavaria JE, Bowen FW, Woo EY, Fairman RM, Pochettino A. The hybrid total arch repair: brachiocephalic bypass and concomitant endovascular aortic arch stent graft placement. J Card Surg. 2007;22:97-102.

E41. Vallejo N, Rodriguez-Lopez JA, Heidari P, Wheatley G, Caparrelli D, Ramaiah V, et al. Hybrid repair of thoracic aortic lesions for zone 0 and 1 in high risk patients. J Vasc Surg. 2012;55:318-25.

E42. Zhou W, Reardon M, Peden EK, Lin PH, Lumsden AB. Hybrid approach to complex thoracic aortic aneurysms in high-risk patients: surgical challenges and clinical outcomes. J Vasc Surg. 2006;44:688-93.

\section{Frozen Elephant Trunk}

E43. Flores J, Kunihara T, Shiiya N, Yoshimoto K, Matsuzaki K, Yasuda K. Extensive deployment of the stented elephant trunk is associated with an increased risk of spinal cord injury. J Thorac Cardiovasc Surg. 2006;131 336-42.

E44. Miyairi T, Kotsuka Y, Ezure M, Ono M, Morota T, Kubota H, et al. Open stentgrafting for aortic arch aneurysm is associated with increased risk of paraplegia. Ann Thorac Surg. 2002;74:83-9.

E45. Mizuno T, Toyama M, Tabuchi N, Sunamori M. Transaortic stented graft implantation for aortic arch aneurysm. Its benefits and risk. Jpn J Thorac Cardiovasc Surg. 2003;51:53-8.

E46. Sakurai K, Usui A, Ueda Y, Akita T, Yoshikawa M, Murayama H, et al. Midterm results for endovascular stent grafts via median sternotomy for distal aortic arch aneurysm. J Artif Organs. 2006;9:149-53.

E47. Uchida N, Shibamura H, Katayama A, Sutoh M, Kuraoka M, Ishihara H. Longterm results of the frozen elephant trunk technique for the extensive arteriosclerotic aneurysm. J Thorac Cardiovasc Surg. 2010;139:913-7.

\section{Stented Elephant Trunk}

E48. Carroccio A, Spielvogel D, Ellozy SH, Lookstein RA, Chin IY, Minor ME Sheahan CM, Teodorescu VJ, Griepp RB, Marin ML. Aortic arch and descending thoracic aortic aneurysms: experience with stent grafting for second-stage "elephant trunk" repair. Vascular. 2005;13:5-10.

E49. Greenberg RK, Haddad F, Svensson L, O’Neill S, Walker E, Lyden SP, Clair D Lytle B. Hybrid approaches to thoracic aortic aneurysms: the role of endovascular elephant trunk completion. Circulation. 2005;112:2619-26.

E50. Kawaharada N, Kurimoto Y, Ito T, Koyanagi T, Yamauchi A, Nakamura M, Takagi N, Higami T. Hybrid treatment for aortic arch and proximal descending thoracic aneurysm: experience with stent grafting for second-stage elephant trunk repair. Eur J Cardiothorac Surg. 2009;36:956-61.

E51. Kieffer E, Koskas F, Cluzel P, Benhamou A, Bahnini A, Chiche L. Endoluminal repair of the aortic arch combined with revascularization of supra-aortic arteries. In: Branchereau A, Jacobs M, eds. EVC 2004. Hybrid vascular procedures. Malden (MA): Blackwell Publishing; 2005:75-84.

E52. Jim J, Moon MR, Rubin BG, Sicard GA, Sanchez LA. Hybrid repair of distal arch aortic aneurysms: endovascular elephant trunk completion. Ann Vasc Surg. 2011;25:598-604

E53. Tse LW, MacKenzie KS, Montreuil B, Obrand DI, Steinmetz OK. The proxima landing zone in endovascular repair of the thoracic aorta. Ann Vasc Surg. 2004, $18: 178-85$.

\section{E-References: Open Arch Repair}

E54. Matalanis G, Hata M, Buxton BF. A retrospective comparative study of deep hypothermic circulatory arrest, retrograde, and antegrade cerebral perfusion in aortic arch surgery. Ann Thorac Cardiovasc Surg. 2003;9:174-9.

E55. Nakai M, Shimamoto M, Yamazaki F, Fujita S, Aoyama A, Chin T, et al. Longterm results after surgery for aortic arch nondissection aneurysm. Kyobu Geka. 2002;55:280-4

E56. Strauch JT, Böhme Y, Franke UF, Wittwer T, Madershahian N, Wahlers T. Selective cerebral perfusion via right axillary artery direct cannulation for aortic arch surgery. Thorac Cardiovasc Surg. 2005;53:334-40.

E57. Sundt TM 3rd, Orszulak TA, Cook DJ, Schaff HV. Improving results of open arch replacement. Ann Thorac Surg. 2008;86:787-96.

E58. Kazui T, Yamashita K, Washiyama N, Terada H, Bashar AH, Suzuki K, et al Aortic arch replacement using selective cerebral perfusion. Ann Thorac Surg. 2007;83:S796-8.

E59. Patel HJ, Nguyen C, Diener AC, Passow MC, Salata D, Deeb GM. Open arch reconstruction in the endovascular era: analysis of 721 patients over 17 years. $J$ Thorac Cardiovasc Surg. 2011;141:1417-23.

E60. Urbanski PP, Lenos A, Bougioukakis P, Neophytou I, Zacher M, Diegeler A Mild-to-moderate hypothermia in aortic arch surgery using circulatory arrest: a change of paradigm? Eur J Cardiothorac Surg. 2012;41:185-91.

\section{E-References: Excluded Studies}

\section{Duplicated Materials}

E61. Bergeron P, Mangialardi N, Costa P, Coulon P, Douillez V, Serreo E, et al. Great vessel management for endovascular exclusion of aortic arch aneurysms and dissections. Eur J Vasc Endovasc Surg. 2006;32:38-45.

E62. Melissano G, Civilini E, Bertoglio L, Calliari F, Setacci F, Calori G, et al. Results of endografting of the aortic arch in different landing zones. Eur J Vasc Endovasc Surg. 2007;33:561-6. 
E63. Melissano G, Civilini E, de Moura MR, Calliari F, Chiesa R. Single center experience with a new commercially available thoracic endovascular graft. Eur J Vasc Endovasc Surg. 2005;29:579-85.

E64. Chiesa R, Melissano G, Marrocco-Trischitta MM, Civilini E, Setacci F. Spinal cord ischemia after elective stent-graft repair of the thoracic aorta. $J$ Vasc Surg. 2005;42:11-7.

E65. Geisbüsch P, Kotelis D, Hyhlik-Dürr A, Hakimi M, Attigah N, Böckler D. Endografting in the aortic arch-does the proximal landing zone influence outcome? Eur J Vasc Endovasc Surg. 2010;39:693-9.

E66. Czerny M, Gottardi R, Zimpfer D, Schoder M, Grabenwoger M, Lammer J, et al. Mid-term results of supraaortic transpositions for extended endovascular repair of aortic arch pathologies. Eur J Cardiothorac Surg. 2007;31:623-7.

E67. Hughes GC, Daneshmand MA, Swaminathan M, Nienaber JJ, Bush EL, Husain AH, et al. "Real world" thoracic endografting: results with the Gore TAG device 2 years after U.S. FDA approval. Ann Thorac Surg. 2008;86: 1530-7.

E68. Hughes GC, Nienaber JJ, Bush EL, Daneshmand MA, McCann RL. Use of custom Dacron branch grafts for "hybrid" aortic debranching during endovascular repair of thoracic and thoracoabdominal aortic aneurysms. $J$ Thorac Cardiovasc Surg. 2008;136:21-8.

E69. Shah AA, Bhattacharya SD, McCann RL, Hughes GC. Pan-aortic hybrid treatment of mega-aorta syndrome. J Vasc Surg. 2011;53:1398-401.

E70. Saleh HM, Inglese L. Combined surgical and endovascular treatment of aortic arch aneurysms. J Vasc Surg. 2006;44:460-6.

E71. Desai ND, Pochettino A, Szeto WY, Moser GW, Moeller PJ, Sodhi N, et al. Thoracic endovascular aortic repair: evolution of therapy, patterns of use, and results in a 10-year experience. J Thorac Cardiovasc Surg. 2011;142: 587-94.

E72. Milewski RK, Szeto WY, Pochettino A, Moser GW, Moeller P, Bavaria JE. Have hybrid procedures replaced open aortic arch reconstruction in high-risk patients? A comparative study of elective open arch debranching with endovascular stent graft placement and conventional elective open total and distal aortic arch reconstruction. J Thorac Cardiovasc Surg. 2010;140:590-7.

E73. Pochettino A, Brinkman WT, Moeller P, Szeto WY, Moser W, Cornelius K, et al. Antegrade thoracic stent grafting during repair of acute DeBakey I dissection prevents development of thoracoabdominal aortic aneurysms. Ann Thorac Surg. 2009;88:482-9.

E74. Pichlmaier MA, Teebken OE, Khaladj N, Weidemann J, Galanski M, Haverich A. Distal aortic surgery following arch replacement with a frozen elephant trunk. Eur J Cardiothorac Surg. 2008;34:600-4.

E75. Liu ZG, Sun LZ, Chang Q, Zhu JM, Dong C, Yu CT, et al. Should the "elephant trunk" be skeletonized? Total arch replacement combined with stented elephant trunk implantation for Stanford type A aortic dissection. J Thorac Cardiovasc Surg. 2006;131:107-13.

\section{E-Reviews}

E76. Antoniou GA, El Sakka K, Hamady M, Wolfe JH. Hybrid treatment of complex aortic arch disease with supra-aortic debranching and endovascular stent graft repair. Eur J Vasc Endovasc Surg. 2010;39:683-90.

E77. Koullias GJ, Wheatley GH 3rd. State-of-the-art of hybrid procedures for the aortic arch: a meta-analysis. Ann Thorac Surg. 2010;90:689-97.

E78. Szeto WY, Bavaria JE. Hybrid repair of aortic arch aneurysms: combined open arch reconstruction and endovascular repair. Semin Thorac Cardiovasc Surg. 2009;21:347-54.

E79. Kotelis D, Geisbüsch P, Attigah N, Hinz U, Hyhlik-Dürr A, Böckler D. Total vs hemi-aortic arch transposition for hybrid aortic arch repair. J Vasc Surg. 2011; 54:1182-6.

E80. Murzi M, Tiwari KK, Farneti PA, Glauber M. Might type A acute dissection repair with the addition of a frozen elephant trunk improve long-term survival compared to standard repair? Interact Cardiovasc Thorac Surg. 2010;11: 98-102.

\section{No Details}

E81. Zipfel B, Hammerschmidt R, Krabatsch T, Buz S, Weng Y, Hetzer R. Stentgrafting of the thoracic aorta by the cardiothoracic surgeon. Ann Thorac Surg. 2007;83:441-8.

E82. Fajer S, Eyal A, Lubezky N, Karmeli R. Combined surgical and endovascular repair of type B thoracic aortic dissecting aneurysm after failed endovascular treatment. Eur J Vasc Endovasc Surg. 2004;27:559-62. 